\title{
Coarse differentiation and quasi-isometries of a class of solvable Lie groups I
}

\author{
IRINE PENG
}

\begin{abstract}
This is the first of two papers that aim to understand quasi-isometries of a class of unimodular split solvable Lie groups. In the present paper, we show that locally (in a coarse sense), a quasi-isometry between two groups in this class is close to a map that respects their group structures. In the sequel to this paper [11], we will use this result to show quasi-isometric rigidity.
\end{abstract}

\section{Introduction}

A $(\kappa, C)$ quasi-isometry $f$ between metric spaces $X$ and $Y$ is a map $f: X \longrightarrow Y$ satisfying

$$
\frac{1}{\kappa} d(p, q)-C \leq d(f(p), f(q)) \leq \kappa d(p, q)+C
$$

with the additional property that there is a number $C$ such that $Y$ is the $C$ neighborhood of $f(X)$. Two quasi-isometries $f, g$ are considered to be equivalent if there is a number $E>0$ such that $d(f(p), g(p)) \leq E$ for all $p \in X$. A class of groups $\mathcal{G}$ is quasiisometrically rigid if any groups quasi-isometric to an element of $\mathcal{G}$ is in $\mathcal{G}$. While it is well-known that finitely generated solvable groups are not quasi-isometrically rigid (see Dyubina [5]), it is often conjectured that the subclass of polycyclic groups form a quasi-isometrically rigid class (see Eskin, Fisher and Whyte [6, Conjecture 1.2]). The first result that support the conjecture was proved by Eskin, Fisher and Whyte on the quasi-isometries of the three dimensional solvable group SOLV. In this two-part paper, we generalize the SOLV results to a class of higher rank solvable Lie groups by generalizing the techniques introduced by Eskin, Fisher and Whyte in $[6 ; 7 ; 8]$.

\subsection{Nondegenerate, split abelian-by-abelian solvable groups}

By a result of Auslander, a connected and simply connected solvable Lie group is any Lie group $\mathcal{L}$ satisfying the sequence

$$
1 \rightarrow \mathcal{U} \rightarrow \mathcal{L} \rightarrow \mathbb{R}^{s} \rightarrow 1,
$$


where $\mathcal{U}$, called the nilradical of $\mathcal{L}$, is its unique maximal normal connected nilpotent subgroup; see Auslander [1].

In a group $G$, an element $x \in G$ is called exponentially distorted if there are numbers $c, \epsilon$ such that for all $n \in \mathbb{Z}$,

$$
\frac{1}{c} \log (|n|+1)-\epsilon \leq\left\|x^{n}\right\|_{G} \leq c \log (|n|+1)+\epsilon,
$$

where $\left\|x^{n}\right\|_{G}$ is the distance between the identity and $x^{n}$ in $G$. Guivarc'h [9] (rediscovered later by Osin [10]) showed that in a connected, simply connected solvable Lie group $\mathcal{L}$, the set of exponentially distorted elements form a normal subgroup, called the exponential radical, inside of the nilradical. We call a solvable Lie group $G$ split if the sequence (1) splits and its exponential radical coincides with its nilradical; abelianby-abelian if in addition, its nilradical is abelian. We can therefore represent a split abelian-by-abelian solvable Lie group $G$ as a semidirect product $\mathbf{H} \rtimes_{\varphi} \mathbf{A}$ where both $\mathbf{H}$ and $\mathbf{A}$ are abelian. The dimension of $\mathbf{A}$ is called the rank of $G$. We say such a group is nondegenerate if every (nontrivial) element of $\varphi(\mathbf{A})$ has at least one eigenvalue whose absolute value is not one. So for example, SOLV is a rank 1 unimodular, nondegenerate, split abelian-by-abelian solvable group.

\subsection{Statement of results}

Associated to nondegenerate, split abelian-by-abelian $G=\mathbf{H} \rtimes_{\varphi} \mathbf{A}$, where $\varphi: \mathbf{A} \longrightarrow$ $\operatorname{Aut}(\mathbf{H})$, is a finite set $\Delta$ of real-valued linear functionals on $\mathbf{A}$, called roots that arise from the simultaneous triangulation of linear maps $\{\varphi(a)\}_{a \in \mathbf{A}}$, and a decomposition of $\mathbf{H}$ into direct sums of subspaces $\left\{V_{\alpha}\right\}_{\alpha \in \Delta}$. (See Section 2.1).

Given a homomorphism $\varphi: \mathbf{A} \rightarrow \operatorname{GL}(n, \mathbb{R})$, the absolute Jordan form of $\varphi$, is a homomorphism $|\varphi|: \mathbf{A} \rightarrow \operatorname{GL}(n, \mathbb{R})$ where $|\varphi|(t), t \in \mathbf{A}$, is the matrix whose entries are absolute values of that of $\varphi(t)$ (see Definition 2.1.1).

Corollary [11, Corollary 5.3.7] If two nondegenerate, unimodular, split abelian-byabelian solvable Lie groups $G=\mathbf{H} \rtimes_{\varphi} \mathbf{A}, G^{\prime}=\mathbf{H}^{\prime} \rtimes_{\varphi^{\prime}} \mathbf{A}^{\prime}$ are quasi-isometric, then there is an isomorphism $f: \mathbf{A} \rightarrow \mathbf{A}^{\prime}$ such that $\varphi$ and $\varphi^{\prime} \circ f$ have the same absolute Jordan form.

We now introduce an equivalence relation on the set of roots: two roots are equivalent if they are positive multiples of each other. Let $[\Xi]$ denote the equivalence class containing the root $\Xi$. In this way we have a coarser decomposition

$$
\mathbf{H}=\bigoplus_{[\alpha]} V_{[\alpha]}
$$


where $V_{[\alpha]}=\bigoplus_{\Xi \in[\alpha]} V_{\Xi}$. We call a left translate of $V_{[\alpha]}$, a $[\alpha]$ horocycle; and by $[\alpha]$ horocycle foliations in $G$ (resp. in $\mathbf{H}$ ) we mean the set of $[\alpha]$ horocycles in $G$ (resp. in $\mathbf{H}$ ). Similarly, for each root class $[\Xi]$, we call a left translate of the kernel of $\Xi$, a $[\Xi]$ kernel leaf; and by $[\Xi]$ kernel foliations in $G$ (resp. in $\mathbf{A}$ ) we mean the set of all $[\Xi]$ kernel leaves in $G$ (resp. in $\mathbf{A}$ ).

A map $\phi: G \rightarrow G^{\prime}$ is standard if it is a product map $f \times g$, where $f: \mathbf{A} \rightarrow \mathbf{A}^{\prime}$, $g: \mathbf{H} \rightarrow \mathbf{H}^{\prime}$ satisfying the following: For some bijection $\sigma$ between root classes of $G$ and root classes of $G^{\prime}$,

- $f$ sends hyperplanes parallel to kernels of $[\Xi]$ to hyperplanes parallel to kernels of $\sigma[\Xi]$;

- $g$ sends foliations by $[\Xi]$ horocycles to foliations by $\left[\sigma_{f}(\Xi)\right]$ horocycles, for each root class $[\Xi]$.

Theorem [11, Theorem 5.3.6] (abridged) Let $G=\mathbf{H} \rtimes_{\varphi} \mathbf{A}, G^{\prime}=\mathbf{H}^{\prime} \rtimes_{\varphi^{\prime}} \mathbf{A}^{\prime}$ be nondegenerate, unimodular, split abelian-by-abelian solvable Lie groups, and $\phi: G \longrightarrow G^{\prime}$ a $(\kappa, C)$ quasi-isometry. Then $\phi$ is bounded distance from a composition of a left translation and a standard map.

Corollary [11, Corollary 1.0.2]

$$
\mathrm{QI}(G)=\left(\prod_{[\alpha]} \operatorname{Bilip}\left(V_{[\alpha]}\right)\right) \rtimes \operatorname{Sym}(G) .
$$

Here $\operatorname{Sym}(G)$ is a finite group, analogous to the Weyl group in reductive Lie groups. It reflects the symmetries of $G$. (See Section 2.1).

We can also distinguish such groups base on the diagonalizability of $\varphi$ in the following.

Corollary [11, Corollary 5.3.7] Let $G=\mathbf{H} \rtimes_{\varphi} \mathbf{A}, G^{\prime}=\mathbf{H}^{\prime} \rtimes_{\varphi^{\prime}} \mathbf{A}^{\prime}$ be nondegenerate, unimodular, split abelian-by-abelian solvable Lie groups. If $\varphi$ is diagonalizable and $\varphi^{\prime}$ is not, then there is no quasi-isometry between them.

Using the work of Dymarz [3] on quasi-similar maps on the boundary of $G$ when $\varphi$ is diagonalizable, its generalization by Dymarz and the author [4], and a theorem of Mostow, we obtain the following.

Corollary [11, Corollary 5.3.9, Corollary 5.3.11] If $\Gamma$ is a finitely generated group quasi-isometric to a unimodular, nondegenerate split abelian-by-abelian $G=\mathbf{H} \rtimes_{\varphi} \mathbf{A}$, then $\Gamma$ is virtually a lattice in a semidirect product of $\mathbf{H}$ with $\mathbf{A}$, so in particular is virtually polycyclic. 
1.2.1 Remark Note that the semidirect product between $\mathbf{H}$ with $\mathbf{A}$ in the corollary above does not have to be isomorphic to $G$, nor does the lattice $\Gamma$ have to commensurate with a subgroup in $G$. Examples showing that this is the best one can do can be constructed by following the idea of Bridson and Gersten [2, Theorem 5.8].

\subsection{Main result in the present paper}

A compact convex set $\Omega \subset \mathbb{R}^{n}$ determines a bounded set $\mathbf{B}(\Omega)$ in $G$ (see Section 2.2). We write $\rho \Omega$ for the compact convex set obtained by scaling $\Omega$ by a factor of $\rho$ from the barycenter of $\Omega$. All the arguments in this paper are local in nature. We state the main result below.

1.3.1 Definition A map $\phi: G \rightarrow G^{\prime}$ is called "standard" if for some bijection $\sigma$, between root classes of $G$ and that of $G^{\prime}, \phi=f \times g$ where $f: \mathbf{A} \rightarrow \mathbf{A}^{\prime}, g: \mathbf{H} \rightarrow \mathbf{H}^{\prime}$ such that for each root class $[\Xi], f$ sends $[\boldsymbol{\Xi}]$ kernel foliations to $\sigma([\boldsymbol{\Xi}])$ kernel foliations, and $g$ sends $[\Xi]$ horocycle foliations to $\sigma([\Xi])$ horocycle foliations.

1.3.2 Remark Note that when $G$ has at least $\operatorname{rank}(G)+1$ many root kernels, the condition on $f$ in Definition 1.3.1 means that $f$ is affine; when $G$ is rank 1 , the condition on $f$ is empty.

1.3.3 Theorem Let $G, G^{\prime}$ be nondegenerate, unimodular, split abelian-by-abelian Lie groups, and $\phi: G \rightarrow G^{\prime}$ be a $(\kappa, C)$ quasi-isometry. Given $0<\theta, \delta<1$, there are numbers $L_{0}, 0<\rho<1$ with the following properties.

If $\Omega \subset \mathbf{A}$ is a product of intervals of equal size at least $L_{0}$, then a tiling of $\mathbf{B}(\Omega)$ by isometric copies of $\mathbf{B}(\rho \Omega)$

$$
\mathbf{B}(\Omega)=\bigsqcup_{j \in \mathbf{J}} \mathbf{B}\left(\omega_{j}\right) \sqcup \Upsilon
$$

contains a subset $\mathbf{J}_{0} \subset \mathbf{J}$ of relative measure at least $1-\theta$, such that for all $j \in \mathbf{J}_{0}$, there is a subset $\mathcal{P}_{0}\left(\omega_{j}\right) \subset \mathcal{P}\left(\omega_{j}\right)$ with relative measure at least $1-\theta$ such that $\left.\phi\right|_{\mathcal{P}_{0}\left(\omega_{j}\right)}$ is within $O\left(\delta \operatorname{diam}\left(\omega_{j}\right)\right)$ of a standard map $g_{j} \times f_{j}$.

Acknowledgements I would like to thank Alex Eskin for his patience and guidance. I also owe much to David Fisher for his help and support.

\section{Preliminaries}

In this section, we first describe the geometry of a class of unimodular solvable Lie group mentioned in the Introduction, followed by a list of notation that will be used in the remainder of this paper. 


\subsection{Geometry of nondegenerate, split abelian-by-abelian groups}

Let $G=\mathbf{A} \ltimes_{\varphi} \mathbf{H}$ be a nondegenerate, split abelian-by-abelian solvable Lie group. Its Lie algebra also admits a semidirect product structure as $\mathfrak{g}=\mathfrak{a} \ltimes_{\bar{\varphi}} \mathfrak{h}$, where $\bar{\varphi}: \mathfrak{a} \rightarrow \operatorname{End}(\mathfrak{h})$. Fix a basis $\mathcal{B}$ of $\mathfrak{h}$ such that for every $\mathbf{t} \in \mathfrak{a}, \bar{\varphi}(\mathbf{t})$ is in real Jordan form, and so $\bar{\varphi}(\mathbf{t})=\delta_{\mathbf{t}}+v_{\mathbf{t}}+\eta_{\mathbf{t}}$ where $\delta_{\mathbf{t}}$ is diagonal, $v_{\mathbf{t}}$ is superdiagonal and $\eta_{\mathbf{t}}$ is skew-symmetric. The entries of $\delta_{t}$ are real-valued linear functionals of $\mathfrak{a}$, and are called roots. For each root $\alpha$, let $V_{\alpha} \subset \mathfrak{h}$ be the subspace on which $\delta_{\mathbf{t}}$ is $\alpha(\mathbf{t})$ times the identity matrix. Requiring the nilradical to coincide with the exponential radical is the same as demanding that the constant function zero is not a root. By identifying $\mathfrak{a}$ with $\mathbf{A}$, and similarly $\mathfrak{h}$ with $\mathbf{H}$ via the exponential maps, we have

$$
\varphi(\mathbf{t})=e^{\delta_{\mathbf{t}}+v_{\mathbf{t}}} e^{\eta_{\mathbf{t}}}
$$

Since $\eta_{\mathbf{t}}$ is skew-symmetric, it follows that $e^{\eta_{\mathbf{t}}}$ is in the compact group $O(\operatorname{dim}(H), \mathbb{R})$. The nondegenerate condition is equivalent to saying that the roots $\operatorname{span} \operatorname{Hom}(\mathfrak{a}, \mathbb{R})$. This also shows that $G$ is unimodular if and only if $\varphi(\mathbf{t})$ has determinant 1 (or that the sum of all the roots is the constant function zero.)

2.1.1 Definition By the absolute Jordan form of $\varphi$, we mean the map $|\varphi|: \mathbf{A} \rightarrow$ $\operatorname{GL}(\operatorname{dim}(\mathbf{H}), \mathbb{R})$ given by

$$
|\varphi|(\mathbf{t})=e^{\delta_{\mathbf{t}}+v_{\mathbf{t}}}
$$

We label the elements of $\mathcal{B}$ as $\left\{e_{i}^{\alpha}\right\}_{\alpha \in \Delta, 1 \leq i \leq n_{\alpha}}$, where $\left\{e_{i}^{\alpha}\right\}_{i}$ is a basis of $V_{\alpha}$. In this way the restriction of $|\varphi|(\mathbf{t})$ to $V_{\alpha}$, when expressed in terms of basis $\mathcal{B} \cap V_{\alpha}$ is the product between the scalar $e^{\alpha(\mathbf{t})}$ and the matrix

$$
N(\mathbf{t})=\left(\begin{array}{cccccc}
1 & u & u^{2} / 2 ! & \cdots & u^{n-1} /(n-1) ! & u^{n} / n ! \\
& 1 & u & \cdots & u^{n-2} /(n-2) ! & u^{n-1} /(n-1) ! \\
& & 1 & \cdots & u^{n-3} /(n-3) ! & u^{n-2} /(n-2) ! \\
& & & \ddots & \vdots & \vdots \\
& & & & 1 & u \\
& & & & &
\end{array}\right)
$$

where $u=\alpha(\mathbf{t}) /|\alpha|$, and $|\alpha|$ is the standard Euclidean norm of $\alpha$.

Fix a basis $\left\{E_{j}\right\}_{j}$ in $\mathbf{A}$ (for example, the duals of a subset of roots), and for each $\mathbf{t} \in \mathbf{A}$, write $\mathbf{t}_{j}$ for its $E_{j}$ coordinate. We coordinatize a point

$$
\left(\sum_{\alpha \in \Delta} \mathbf{x}_{\alpha}\right)(\mathbf{t})=\left(\sum_{\alpha \in \Delta} \sum_{i=1}^{n_{\alpha}} x_{i, \alpha} e_{i}^{\alpha}\right)(\mathbf{t}) \in \mathbf{H} \rtimes_{\varphi} \mathbf{A},
$$


by the $\operatorname{dim}(G)$-tuple of numbers $\left(\left(x_{i, \alpha}\right)_{i, \alpha},\left(\mathbf{t}_{j}\right)\right) \in \mathbb{R}^{\operatorname{dim}(G)}$. In this coordinate system, a left invariant Riemannian metric at $\left(\left(\mathbf{x}_{\alpha}\right)_{\alpha},\left(\mathbf{t}_{j}\right)_{j}\right)$ is

$$
\sum_{j} d\left(\mathbf{t}_{j}\right)^{2}+\sum_{\alpha \in \Delta} e^{-2 \alpha(\mathbf{t})} \sum_{i=1}^{\alpha_{i}}\left(d x_{i, \alpha}+\sum_{\iota=i+1}^{n_{\alpha}} P_{i, \iota}^{\alpha}(\alpha(-\mathbf{t})) d x_{\iota, \alpha}\right)^{2},
$$

where $P_{i, \iota}^{\alpha}$ is a polynomial with no constant term. We see that the above Riemannian metric is bilipschitz to the left invariant Finsler metric

$$
|d \mathbf{t}|+\sum_{\alpha \in \Delta} e^{-\alpha(\mathbf{t})} \sum_{i=1}^{n_{\alpha}}\left(1+Q_{i, \alpha}(|\alpha(-\mathbf{t})|)\right)\left|d x_{i, \alpha}\right|,
$$

where $|d \mathbf{t}|$ means $\sum_{j}\left|d \mathbf{t}_{j}\right|$, and $Q_{i, \alpha}$ is a polynomial with no constant term. In particular when the split abelian-by-abelian solvable group has just one root $a>0$, the Finsler metric given above becomes the following, where $P_{i}$ is a polynomial with no constant term:

$$
|d t|+e^{-a t} \sum_{i}\left(1+P_{i}(a t)\right)\left|d x_{i}\right| .
$$

Rank one abelian-by-abelian solvable groups with nonzero roots will feature quite prominently in this paper, so we now make it a definition.

2.1.2 Definition We label a group of the form $\mathbb{R}^{s} \ltimes_{\psi} \mathbb{R}$, where $\psi$ gives rise to a single nonzero root, by $H_{s+1}$.

2.1.3 Remark The space $H_{s+1}$ defined above is just a slight generalization of the usual hyperbolic plane. In particular, when $\psi$ is diagonal, $H_{s+1}$ is the $s+1$ dimensional hyperbolic space (after some change of variables).

2.1.4 Lemma If $G$ is a nondegenerate, split abelian-by-abelian, then it can be quasiisometrically embedded into $\prod_{\alpha \in \triangle} H_{\operatorname{dim}\left(V_{\alpha}\right)+1}$.

Proof Consider the map $G \longrightarrow \prod_{\alpha \in \Delta} H_{\operatorname{dim}\left(V_{\alpha}\right)+1}$ sending $\left(\left(\mathbf{x}_{\alpha}\right)_{\alpha}, \mathbf{t}\right)$ to $\left(\mathbf{x}_{\alpha}, \alpha(\mathbf{t})\right)_{\alpha}$. The claim now follows from the inequality below.

$$
\begin{aligned}
\frac{1}{|\triangle|} \sum_{\alpha \in \Delta}(|d \alpha(\mathbf{t})| & \left.+e^{-\alpha(\mathbf{t})} \sum_{i=1}^{n_{\alpha}}\left(1+Q_{i, \alpha}(|\alpha(-\mathbf{t})|)\right)\left|d x_{i, \alpha}\right|\right) \\
& \leq|d \mathbf{t}|+\sum_{\alpha \in \Delta} e^{-\alpha(\mathbf{t})} \sum_{i=1}^{n_{\alpha}}\left(1+Q_{i, \alpha}(|\alpha(-\mathbf{t})|)\right)\left|d x_{i, \alpha}\right| \\
& \leq \sum_{\alpha \in \Delta}\left(|d \alpha(\mathbf{t})|+e^{-\alpha(\mathbf{t})} \sum_{i=1}^{n_{\alpha}}\left(1+Q_{i, \alpha}(|\alpha(-\mathbf{t})|)\right)\left|d x_{i, \alpha}\right|\right) .
\end{aligned}
$$


Recommendation to the reader The reader can assume $\varphi$ is diagonal on first reading, because the geometry of a general $H_{s+1}$ is qualitatively the same as that of a hyperbolic space, as we will see in the next paragraph.

To understand the geometry of $H_{s+1}$ better, we can assume without loss of generality that the root $a$ is the constant function 1, so the Finsler metric in (2) becomes $d t+e^{-t} Q(t) d \mathbf{x}$ for some polynomial $Q(t)$. Since exponential grows faster than polynomials, for any large positive number $x$, there is a $t_{0}$ such that $e^{-t} Q(t) x \leq 1$ for all $t \geq t_{0}$. So a function quasi-isometric to the distance function on $H_{S+1}$ is the following:

$$
d\left(\left(\mathbf{x}_{1}, t_{1}\right),\left(\mathbf{x}_{2}, t_{2}\right)\right)= \begin{cases}\left|t_{1}-t_{2}\right|+1 & \text { if } e^{-t_{i}} Q\left(t_{i}\right)\left|\mathbf{x}_{1}-\mathbf{x}_{2}\right| \leq 1 \\ \left|T-t_{1}\right|+\left|T-t_{2}\right|+1 & \text { otherwise, }\end{cases}
$$

where $T=U\left(\left|\mathbf{x}_{1}-\mathbf{x}_{2}\right|\right)$ is the smallest value of $t$ such that

$$
e^{-t} Q(t)\left|\mathbf{x}_{1}-\mathbf{x}_{2}\right| \leq 1 \text {. }
$$

Furthermore, the following relation

$$
\frac{1}{e^{t}}<\frac{Q(t)}{e^{t}}<\frac{e^{(1 / 2) t}}{e^{t}} \text { for } t \text { sufficiently large }
$$

and the fact that both $e^{-t}$ and $Q(t) e^{-t}$ are decreasing functions when $t$ becomes big enough means that we have the following inequalities for the inverses of $e^{t}$ and $e^{t} / Q(t)$ :

$$
\ln (x)-C_{Q} \leq U_{Q}(x) \leq 2 \ln (x)+C_{Q} \text { for } x>1
$$

for some constant $C$ depending only on the polynomial $Q$.

A left translate of $\mathbf{A}$, or a subset of it, is called a flat. For two points $p, q \in \mathbf{H}$ with coordinates $\left(\mathbf{x}_{\alpha}\right)_{\alpha \in \Delta}$ and $\left(\mathbf{y}_{\alpha}\right)_{\alpha \in \Delta}$, we call the subsets of $p \mathbf{A}$ and $q \mathbf{A}$ that are within distance $O(1)$ of each other, the coarse intersection between $p \mathbf{A}$ and $q \mathbf{A}$.

According to the embedded metric in Lemma 2.1.4, the coarse intersection of $p \mathbf{A}$ and $q \mathbf{A}$ is the $p$ and $q$ translates of the following subset of $\mathbf{A}$ :

$$
\bigcap_{\alpha \in \Delta: \ln \left(\left|\mathbf{x}_{\alpha}-\mathbf{y}_{\alpha}\right|\right) \geq 1} \alpha^{-1}\left[U_{\alpha}\left(\left|\mathbf{x}_{\alpha}-\mathbf{y}_{\alpha}\right|\right), \infty\right] .
$$

As the roots sum up to zero in a nondegenerate, unimodular, split abelian-by-abelian group, the coarse intersection of two flats can be empty. If it is not empty, then the equation above says that it is a convex subset of $\mathbf{A}$ bounded by hyperplanes parallel to root kernels. Often we will say that $p \mathbf{A}$ and $q \mathbf{A}$ come together along this subset of $\mathbf{A}$. 


\subsection{Notation}

\subsubsection{General remarks about paths, neighborhoods}

Division of a curve By scale we mean a number $0<\rho \leq 1$. We will often examine a quasi-geodesic on different scales, and see if the quasi-geodesic on that scale satisfies certain properties. This roughly means that we subdivide the quasi-geodesic into subsegments whose lengths are $\rho$ proportion of the length of the original one, and see if each one of them has the desired properties.

In practice, instead of dealing with length, we use distance between end points of a curve. Let $\zeta:[a, b] \rightarrow Y$ be a mapping (not necessarily continuous).

- Choose $r>0$, we can divide the image of $\zeta$ into subcurves whose end points are $r$ apart. That is, $\hat{\mathcal{S}}(\zeta, r)=\left\{q_{i}\right\}_{i=1}^{n_{r}}$, is the set of dividing points on $\zeta$, where $q_{0}=\zeta(a), q_{n_{r}}=\zeta(b)$, and

$$
\zeta^{-1}\left(q_{i+1}\right)=\min \left\{t \geq \zeta^{-1}\left(q_{i}\right) \mid d\left(\zeta(t), q_{i}\right)=r\right\} .
$$

We define the $r$ length of $\zeta$ to be

$$
\|\zeta\|_{r}:=\sum_{i} d\left(q_{i}, q_{i+1}\right)
$$

- Given two points $p, q \in \zeta$, we write $\zeta_{[p, q]}$ for the part of $\zeta$ between $p$ and $q$. Define $\mathcal{S}(\zeta, r)=\left\{\zeta_{\left[q_{i}, q_{i+1}\right]}\right\}$, to be the set of subcurves after division by $\hat{\mathcal{S}}(\zeta, r)$.

- Let $\mathbf{P}$ be a statement. Define $\mathcal{S}(\zeta, r, \mathbf{P})=\left\{\zeta^{i} \in \mathcal{S}(\zeta, r) \mid \zeta^{i}\right.$ satisfies $\left.\mathbf{P}\right\}$ to be those subcurves satisfying the statement $\mathbf{P}$.

2.2.2 Definition We say a mapping $\zeta:[a, b] \rightarrow Y$ (not necessarily continuous) is a $(\kappa, C)$ coarsely rectifiable curve if for all $r_{1}, r_{2} \geq C$,

$$
\frac{\|\zeta\|_{r_{1}}}{\|\zeta\|_{r_{2}}} \leq \kappa .
$$

We will often use $\|\zeta\|$ to denote $\|\zeta\|_{C}$ and call it the coarse length of $\zeta$ if it is clear from the context what the implied $C$ is.

2.2.3 Remark A quasi-geodesic is an example of a coarsely rectifiable curve. 
Neighborhoods We write $B(p, r)$ for the ball centered at $p$ of radius $r$, and $N_{c}(A)$ for the $c$ neighborhood of the set $A$. We also write $d_{H}(A, B)$ for the Hausdorff distance between two sets $A$ and $B$. If $\Omega \subset \mathbb{R}^{k}$ is a bounded compact set, and $r \in \mathbb{R}$, we write $r \Omega$ for the bounded compact set that is scaled from $\Omega$ with respect to the barycenter of $\Omega$. Given a set $X$, a point $x_{0} \in X$, the $(\eta, C)$ linear neighborhood of $X$ with respect to $x_{0}$ consists of points $y$ such that there is a $\hat{x} \in X$ with $d(y, \hat{x})=$ $\left.d(y, X) \leq \eta d\left(\hat{x}, x_{0}\right)+C\right\}$. If a quasi-geodesic $\lambda$ is within the $(\eta, C)$ linear (or just $\eta$-linear) neighborhood of a geodesic segment $\gamma$, where $\eta \ll 1$ and $C \ll \eta|\lambda|$, then we say that $\lambda$ admits a geodesic approximation by $\gamma$.

\subsubsection{Notation used in split abelian-by-abelian groups}

Subspaces of $G$ Let $G=\mathbf{H} \rtimes \mathbf{A}$ stands for a nondegenerate, split abelian-by-abelian group. Recall that $\triangle$ is the set of roots. Fix a point $p \in G$. We define the following:

- For $\alpha \in \triangle$ a root, we write $\vec{v}_{\alpha} \in \mathfrak{a}$ with norm 1 with respect to the usual Euclidean metric such that with respect to the usual Euclidean inner product $\langle\cdot$,$\rangle , the$ functional $\left\langle\vec{v}_{\alpha}, \cdot\right\rangle$ is a positive multiple of $\alpha$. Note that $\vec{v}_{\alpha}$ is well-defined as a function of root class $[\alpha]$.

- Given $\vec{v} \in \mathbf{A}$, the subgroup $G_{\vec{v}}=\mathbf{H} \rtimes \mathbb{R}<G=\mathbf{A} \ltimes_{\varphi} \mathbf{H}$ given by $t \mathbf{H} t^{-1}=\varphi(t \vec{v}) \mathbf{H}$, has a natural flow which allows for a $\mathbf{H}$ left-invariant splitting on the tangent bundle of $\mathbf{H}$

$$
T_{e} \mathbf{H}=E_{\vec{v}}^{+} \oplus E_{\vec{v}}^{0} \oplus E_{\vec{v}}^{-}
$$

be defined as follows.

$$
\begin{aligned}
& W_{\vec{v}}^{+}=\bigoplus_{\Xi(\vec{v})>0} V_{\Xi}=\operatorname{Exp}\left(E_{\vec{v}}^{+}\right) \\
& W_{\vec{v}}^{-}=\bigoplus_{\Xi(\vec{v})<0} V_{\Xi}=\operatorname{Exp}\left(E_{\vec{v}}^{-}\right) \\
& W_{\vec{v}}^{0}=\bigoplus_{\Xi(\vec{v})=0} V_{\Xi}=\operatorname{Exp}\left(E_{\vec{v}}^{0}\right)
\end{aligned}
$$

If $W_{\vec{v}}^{0}$ is not trivial, then we say the vector $\vec{v}$ is singular. We call left translates of $W_{\vec{v}}^{+}, W_{\vec{v}}^{0}$ and $W_{\vec{v}}^{-}$, respectively, the expanding/unstable, neutral, and contracting/stable leaves of $G_{\vec{v}}$.

- Let $\pi_{A}: G=\mathbf{H} \rtimes \mathbf{A} \longrightarrow \mathbf{A}$ be the projection onto the $\mathbf{A}$ factor as $(\mathbf{x}, \mathbf{t}) \mapsto \mathbf{t}$.

- A context in which the space $H_{s+1}$ in Definition 2.1.2 arises naturally in our group $G$ is the following. For each root $\alpha$, let $\vec{v}_{\alpha}$ be its dual. We label the semidirect product between $\left\langle\vec{v}_{\alpha}\right\rangle$ and $V_{\alpha}$, the generalized eigenspace corresponding to $\alpha$, as $\mathbb{H}_{\alpha}$. There is a natural projection map $\pi_{\alpha}$ : $\longrightarrow \longrightarrow \mathbb{H}_{\alpha}$ as $\left(\mathbf{x}_{1}, \mathbf{x}_{2}, \ldots, \mathbf{x}_{|\Delta|}\right) \mathbf{t} \mapsto\left(\mathbf{x}_{i}, \alpha(\mathbf{t}) \vec{v}_{\alpha}\right)$. 
Boxes and associated objects For $\alpha \in \triangle$, let $b(r) \subset V_{\alpha}$ be a product of intervals of size $2 r$ centered at the origin, ie $[-r, r]^{\operatorname{dim}\left(V_{\alpha}\right)}$. Let $\Omega \subset \mathbf{A}$ be a convex compact set with nonempty interior. We call a subset $\Omega \subset \mathbf{A}$ rounded if for every root $\alpha$

$$
\frac{\left|\operatorname{Proj}_{\text {ker }_{\alpha}}(\Omega)\right|}{|\Omega|} \leq \frac{|\triangle|}{\operatorname{diam}(\Omega)} .
$$

For example, a metric ball in $\mathbf{A}$ is rounded. For a rounded compact convex set $\Omega \subset \mathbf{A}$ centered at the origin, we define $\mathbf{B}(\Omega)$, the box associated to $\Omega$, as the union of left translates of $\Omega$ over all elements of $\left(\prod_{j=1}^{|\Delta|} b\left(e^{\max \left(\alpha_{j}(\Omega)\right)}\right)\right)$. In other words,

$$
\mathbf{B}(\Omega)=\left(\prod_{j=1}^{|\Delta|} b\left(e^{\max \left(\alpha_{j}(\Omega)\right)}\right)\right) \Omega .
$$

2.2.5 Remark A box $\mathbf{B}(\Omega)$ as defined above is just a union of left translates of $\Omega \subset \mathbf{A}$ by a subset of $\mathbf{H}$ whose size happens to be determined by $\Omega$. The size of this subset was chosen so that a large proportion of points in the box $\mathbf{B}(\Omega)$ lie on a quadrilateral (see Definition 4.1.5). In the definition above we have defined this subset of $\mathbf{H}$ as a product of intervals, but this is just a choice of convenience.

Associate to the box $\mathbf{B}(\Omega)$, we use the following notation.

- $\mathcal{L}(\Omega)$ is the set of geodesics in $\mathbf{B}(\Omega)$ whose $\pi_{A}$ images begin and end at points of $\partial \Omega$ such that the ratio between its length and the diameter of $\Omega$ lies in the interval $[1 / m, 1]$. We fix the value of $m$ once $\Omega$ is chosen, and will often omit the $m$ in the expression.

- For $i=2,3, \ldots, n$, write $\mathcal{L}_{i}(\Omega)$ for the set of $i$ dimensional hyperplanes (see bullet point under "Subspaces in $G$ ") in $\mathbf{B}(\Omega)$ such that the ratio between its diameter and the diameter of $\Omega$ lies in the interval $\left[1 / m_{i}, 1\right]$.

- $\mathcal{P}(\Omega)($ or $\mathcal{P}(\mathbf{B}(\Omega)))$ is the set of points in $\mathbf{B}(\Omega)$.

- Let $S$ be an element of $\bigcup_{i=2}^{n} \mathcal{L}_{i}(\Omega) \cup \mathcal{L}(\Omega) \cup \mathcal{P}(\Omega)$. We write $L(S), L_{i}(S)$ for the subset of $\mathcal{L}(\Omega), \mathcal{L}_{i}(\Omega)$ contained or containing $S$, and $P(S)$ for the subset of $\mathcal{P}(\Omega)$ contained in $S$.

2.2.6 Remark Typically we will work with a representative of a quasi-isometry class defined for particular choices of nets. Since any two nets in a space are bounded distance apart, all the coarse arguments remains valid for that entire equivalence. Let $\hat{p}$ : $G \rightarrow X$ assigns $x \in G$, a closest net point. In this way we tend to think of a set $K \subset G$ as a subset of the net $X$ via the identification of $K$ and $\hat{p}(K)$. In particular, 
all the objects in a box defined by bullets points above are finite sets to us, and we equip a box with a counting measure. By abuse of notation, we denote this counting measure by the absolute value sign $|\cdot|$.

2.2.7 Lemma Let $G=\mathbf{H} \rtimes \mathbf{A}$ be a nondegenerate, unimodular, split abelian-byabelian Lie group. Let $\Omega \subset \mathbf{A}$ be a rounded compact convex set with nonempty interior. Then, $\mathbf{B}(r \Omega), r \rightarrow \infty$ is a Fölner sequence. The volume ratio between $N_{\epsilon}(\partial(\mathbf{B}(r \Omega)))$ and $\mathbf{B}(r \Omega)$ is $O(\epsilon / \operatorname{diam}(\mathbf{B}(r \Omega))$.

Proof For each root $\alpha_{j}$, write $\alpha_{j}(\Omega)=\left[b_{j}, a_{j}\right]$. Since the sum of roots is zero, the volume element is $\wedge_{j} d \mathbf{x}_{j} \wedge d \mathbf{t}$. Therefore $\operatorname{vol}(\mathbf{B}(r \Omega))=\left(\prod_{j} e^{r a_{j}}\right) r^{n}|\Omega|$. On the other hand, the area of the boundary is

$$
\left|\partial\left(\prod_{j}\left[0, e^{r a_{j}}\right](r \Omega)\right)\right|=\underbrace{\left|\partial\left(\prod_{j}\left[0, e^{r a_{j}}\right]\right)(r \Omega)\right|}+\underbrace{\left|\left(\prod_{j}\left[0, e^{r a_{j}}\right]\right) \partial(r \Omega)\right|} .
$$

Since box $\mathbf{B}(\Omega)$ is the product of a set in $\mathbf{H}$ with $\Omega$ in $\mathbf{A}$, the boundary of $\mathbf{B}$ consist of the boundary of the set in $\mathbf{H}$ with $\Omega$, whose area is the term labeled (1), and the product of the set in $\mathbf{H}$ with the boundary of $\Omega$, whose area is the term labeled (2).

We estimate the size of each term.

(2) : $\left|\left(\prod_{j}\left[0, e^{r a_{j}}\right]\right) \partial(r \Omega)\right|=\left(\prod_{j} e^{r a_{j}}\right) r^{n-1}|\partial \Omega|$,

(1) : $\left|\partial\left(\prod_{j}\left[0, e^{r a_{j}}\right]\right)(r \Omega)\right|$

$$
\begin{aligned}
& =2 \sum_{j} \int_{\mathbf{t} \in r \Omega} \underbrace{\int_{\mathbf{x}_{1} \in b\left(e^{r a_{1}}\right), \ldots, \mathbf{x}_{i} \in b^{e r a_{i}}} e^{-\alpha_{1}(\mathbf{t})} d \mathbf{x}_{1} \cdots e^{-\alpha_{i}(\mathbf{t})} d \mathbf{x}_{i} \cdots d}_{i \neq j} d \mathbf{t} \\
& =2 \sum_{j}\left(\prod_{i \neq j} e^{r a_{i}} \int_{\mathbf{t} \in r \Omega} e^{\alpha_{j}(\mathbf{t})} d \mathbf{t}\right) \\
& \leq 2 \sum_{j}\left(\prod_{i \neq j} e^{r a_{i}}\left(e^{r a_{j}}-e^{r b_{j}}\right)\left|\operatorname{Proj}_{\operatorname{ker}\left(\alpha_{j}\right)}(r \Omega)\right|\right)
\end{aligned}
$$




$$
\begin{aligned}
& =2\left(\prod_{i} e^{r a_{i}}\right) r^{n-1}\left(\sum_{j}\left|\operatorname{Proj}_{\operatorname{ker}\left(\alpha_{j}\right)}(\Omega)\right|\left(1-e^{-\left(r a_{j}-r b_{j}\right)}\right)\right) \\
& \leq 2|\triangle|\left(\prod_{i} e^{r a_{i}}\right) r^{n-1} \max _{j}\left|\operatorname{Proj}_{\operatorname{ker}\left(\alpha_{j}\right)}(\Omega)\right|,
\end{aligned}
$$

where we used unimodularity in the second equality. Since $\Omega$ is rounded,

$$
\begin{aligned}
\frac{\left|\partial\left(\prod_{j}\left[0, e^{r a_{j}}\right](r \Omega)\right)\right|}{|\mathbf{B}(r \Omega)|} & =\frac{(1)}{\left(\prod_{j} e^{r a_{j}}\right) r^{n}|\Omega|}+\frac{(2)}{\left(\prod_{j} e^{r a_{j}}\right) r^{n}|\Omega|} \\
& \leq \frac{1}{r} \frac{|\partial \Omega|}{|\Omega|}+2|\triangle| \frac{1}{r} \frac{\max _{j}\left|\operatorname{Proj}_{\operatorname{ker}\left(\alpha_{j}\right)}(\Omega)\right|}{|\Omega|} \\
& =O\left(\frac{1}{r} \frac{1}{\operatorname{diam}(\Omega)}\right)=O(1 / \operatorname{diam}(\mathbf{B}(r \Omega))) .
\end{aligned}
$$

\section{Quasi-geodesics}

The purpose of this section is to prove the following.

Theorem 3.5.1 Let $G, G^{\prime}$ be nondegenerate, unimodular, split abelian-by-abelian Lie groups, and $\phi: G \rightarrow G^{\prime}$ be a $(\kappa, C)$ quasi-isometry. Given $0<\theta, \delta<1$, there are numbers $L_{0}, 0<\rho<1$ with the following properties.

If $\Omega \subset \mathbf{A}$ is a product of intervals of equal size at least $L_{0}$, then a tiling of $\mathbf{B}(\Omega)$ by isometric copies of $\mathbf{B}(\varrho \Omega)$

$$
\mathbf{B}(\Omega)=\bigsqcup_{j \in \mathbf{J}} \mathbf{B}\left(\omega_{j}\right) \sqcup \Upsilon
$$

contains a subset $\mathbf{J}_{0} \subset \mathbf{J}$ with a relative measure at least $1-\theta$ such that for all $j \in \mathbf{J}_{0}$,

(i) there is a subset $\mathcal{L}_{0}\left(\omega_{j}\right) \subset \mathcal{L}\left(\omega_{j}\right)$ with relative measure least $1-\theta$, such that their images under $\phi$ are within $\delta$ linear neighborhood of geodesic segments;

(ii) there is a subset $\mathcal{P}_{0}\left(\omega_{j}\right) \subset \mathcal{P}\left(\omega_{j}\right)$ with relative measure at least $1-\theta$, such that for every $p \in \mathcal{P}_{0}\left(\omega_{j}\right)$, at least $1-\theta$ proportion of geodesics passing within $O\left(\delta \operatorname{diam}\left(\omega_{j}\right)\right)$ of $x$, belong to $\mathcal{L}_{0}\left(\omega_{j}\right)$.

In the next few sections we will collect the necessary lemmas and prove the above theorem in Section 3.5. 


\subsection{Quasi-geodesics in nondegenerate, split abelian-by-abelian groups}

In this section, $G$ denotes a nondegenerate, split abelian-by-abelian group. By Lemma 2.1.4, we can use the embedded metric on $G$. We will use the metric property of these $H_{s+1}$ spaces to obtain the following proposition, which says that if a quasi-geodesic in $G$ is long, then its projection in $\mathbf{A}$ (its image under $\pi_{A}$ ) has to be long as well.

3.1.1 Proposition Let $\zeta:[0, L] \rightarrow G$ be a $(\kappa, C)$ quasi-geodesic segment. Suppose $\left\{\pi_{A}(\zeta(t))\right\}$ lies in a ball of diameter $s$. Then for any $p, q \in \zeta, d(p, q) \leq \hbar s$, where $\hbar$ is a constant that depends only the values of $\kappa, C$ and the number of roots.

3.1.2 Corollary (Assumptions as in Proposition 3.1.1) If there are two points $p, q$ on $\zeta$ such that $d(p, q)>\hbar s$, then there is a point $r \in\left[\zeta^{-1}(p), \zeta^{-1}(q)\right]$ such that $d\left(\pi_{A}(p), \pi_{A}(\zeta(r))\right)>s$.

To prove Proposition 3.1.1, we need the following two lemmas whose verifications can be found in the Appendix. In $H_{n^{\prime}+1}=\mathbb{R}^{n^{\prime}} \rtimes \mathbb{R}$, we write $h$ for the projection onto the $\mathbb{R}$ factor.

3.1.3 Lemma Let $\eta:[a, b] \rightarrow H_{n^{\prime}+1}$ be a continuous path such that

- the image of $h \circ \eta$ is contained in an interval of length no bigger than $s$, where $s>\kappa\left(C_{n^{\prime}}\right)^{2}(>2)$ and $C_{n^{\prime}}$ is a constant depending only $H_{n^{\prime}+1}$ (as in (4));

- whenever $i_{1} \leq i_{2} \leq \cdots \leq i_{n} \in[a, b]$,

$$
\frac{\sum_{j} d\left(\eta\left(i_{j}\right), \eta\left(i_{j+1}\right)\right)}{d\left(\eta\left(i_{1}\right), \eta\left(i_{n}\right)\right)} \leq 2 \kappa .
$$

Then, for any $p, q \in \eta([a, b]), d(p, q) \leq \widehat{C}(2 \kappa) s$, where $\widehat{C}$ depends only on $C_{n^{\prime}}$.

Proof See the Appendix.

3.1.4 Lemma Let $a, b \geq 0, A, B>0$. Suppose

$$
\frac{a+b}{A+B}=c_{\alpha} \frac{a}{A}+c_{\beta} \frac{b}{B},
$$

with $c_{\alpha}+c_{\beta}=1$. If $c_{\alpha} \geq c_{\beta}$, then $A \geq B$.

Proof See the Appendix. 
Proof of Proposition 3.1.1 We proceed by induction on the number of roots. The base step where there is just one root is Lemma 3.1.3. Since $\zeta$ is a $(\kappa, c)$ quasi-geodesic, for any $i_{0} \leq i_{1} \leq i_{2} \leq i_{3} \leq \cdots \leq i_{n} \in[0, L]$, we must have

$$
\frac{\sum_{j} d\left(\zeta\left(i_{j}\right), \zeta\left(i_{j+1}\right)\right)}{d\left(\zeta\left(i_{0}\right), \zeta\left(i_{n}\right)\right)} \leq 2 \kappa
$$

We recall from Lemma 2.1 .4 that $d(\cdot, \cdot)=\sum_{l=1}^{|\Delta|} d^{\alpha_{l}}\left(\pi_{\alpha_{l}}(\cdot), \pi_{\alpha_{l}}(\cdot)\right)$, and proceed to simplify Equation (5) by writing $d^{\alpha_{l}}\left(\pi_{\alpha_{l}}\left(\zeta\left(i_{j}\right)\right), \pi_{\alpha_{l}} \zeta\left(i_{j+1}\right)\right)$ as $d_{j}^{\alpha_{l}}$, and $d^{\alpha_{l}}\left(\pi_{\alpha_{l}}\left(\zeta\left(i_{0}\right)\right), \pi_{\alpha_{l}}\left(\zeta\left(i_{n}\right)\right)\right)$ as $d^{\alpha_{l}}$. Now (5) becomes

$$
\frac{\sum_{j}\left(d_{j}^{\alpha_{1}}+d_{j}^{\alpha_{2}}+\cdots+d_{j}^{\alpha_{|\triangle|}}\right)}{d^{\alpha_{1}}+d^{\alpha_{2}}+\cdots+d^{\alpha_{|\triangle|}}} \leq 2 \kappa
$$

Suppose for some weight, say $\alpha_{1}$, we have

$$
\begin{aligned}
& \frac{\sum_{j} d_{j}^{\alpha_{1}}+\sum_{j}\left(d_{j}^{\alpha_{2}}+d_{j}^{\alpha_{3}}+\cdots+d_{j}^{\alpha_{|\triangle|}}\right)}{d^{\alpha_{1}}+\left(d^{\alpha_{2}}+d^{\alpha_{3}}+\cdots+d^{\alpha_{|\triangle|}}\right)} \\
& \quad=c_{\alpha} \frac{\sum_{j} d_{j}^{\alpha_{1}}}{d^{\alpha_{1}}}+c_{\beta} \frac{\sum_{j}\left(d_{j}^{\alpha_{2}}+d_{j}^{\alpha_{3}}+\cdots+d_{j}^{\alpha_{|\triangle|}}\right)}{\left(d^{\alpha_{2}}+d^{\alpha_{3}}+\cdots+d^{\left.\alpha_{|\triangle|}\right)}\right.},
\end{aligned}
$$

where $c_{\alpha}+c_{\beta}=1$, and $c_{\alpha} \geq c_{\beta}$. Therefore $c_{\alpha} \geq 1 / 2$. Since Equation (6) is bounded above by $2 \kappa$, we now have an upper bound for the first term:

$$
\frac{1}{2} \frac{\sum_{j} d_{j}^{\alpha_{1}}}{d^{\alpha_{1}}} \leq 2 \kappa
$$

That is, $\left\{\pi_{\alpha_{1}}\left(\zeta\left(i_{j}\right)\right)\right\} \subset \mathbb{H}_{\alpha_{1}}$ are points whose heights lie in an interval of width no bigger than $s$ (since $\pi_{A}\left(\zeta\left(i_{j}\right)\right)$ lies in a ball of diameter $s$ ), so

$$
\frac{\sum_{j} d^{\alpha_{1}}\left(\pi_{\alpha_{1}}\left(\zeta\left(i_{j}\right)\right), \pi_{\alpha_{1}}\left(\zeta\left(i_{j+1}\right)\right)\right)}{d^{\alpha_{1}}\left(\pi_{\alpha_{1}}\left(\zeta\left(i_{0}\right)\right), \pi_{\alpha_{1}}\left(\zeta\left(i_{n}\right)\right)\right)} \leq 4 \kappa
$$

By Lemma 3.1.3,

$$
d^{\alpha_{1}}\left(\pi_{\alpha_{1}}\left(\zeta\left(i_{0}\right)\right), \pi_{\alpha_{1}}\left(\zeta\left(i_{n}\right)\right)\right) \leq \widehat{C}(4 \kappa) s
$$

Since $c_{\alpha} \geq c_{\beta}$, Lemma 3.1 .4 says $d^{\alpha_{1}} \geq \sum_{l=2}^{|\Delta|} d^{\alpha_{l}}$, which makes

$$
d\left(\zeta\left(i_{0}\right), \zeta\left(i_{n}\right)\right)=d^{\alpha_{1}}+\sum_{l=2}^{|\Delta|} d^{\alpha_{l}} \leq 2 \widehat{C}(4 \kappa) s=2^{2} \widehat{C}(2 \kappa) s
$$


If the first possibility (6) doesn't occur, then for every weight $\alpha_{i^{\prime}}$, we must have

$$
\begin{aligned}
& \frac{\sum_{j}\left(d_{j}^{\alpha_{1}}+d_{j}^{\alpha_{2}} \cdots d_{j}^{\alpha^{|\Delta|}}\right)}{d^{\alpha_{1}}+d^{\alpha_{2}}+\cdots+d^{\alpha_{|\Delta|}}} \\
& \quad=c_{\alpha_{i^{\prime}}} \frac{\sum_{j} d_{j}^{\alpha_{i^{\prime}}}}{d^{\alpha_{i^{\prime}}}}+c_{\beta_{i^{\prime}}} \frac{\sum_{j}\left(d_{j}^{\alpha_{1}}+d_{j}^{\alpha_{2}} \cdots d_{j}^{\alpha_{i^{\prime}-1}}+d_{j}^{\alpha_{i^{\prime}+1}} \cdots d_{j}^{\alpha_{|\Delta|}}\right)}{\sum_{l \neq i^{\prime}} d^{\alpha_{l}}}
\end{aligned}
$$

with $c_{\alpha_{i^{\prime}}}, c_{\beta_{i^{\prime}}} \geq 0, c_{\alpha_{i^{\prime}}}+c_{\beta_{i^{\prime}}}=1$, but $c_{\alpha_{i^{\prime}}} \leq c_{\beta_{i^{\prime}}}$. We fix such an $i^{\prime}$. Then $c_{\beta_{i^{\prime}}} \geq 1 / 2$. Since (7) is bounded above by $2 \kappa$, we obtain an upper bound for the second term:

$$
\frac{\sum_{j}\left(d_{j}^{\alpha_{1}}+d_{j}^{\alpha_{2}} \cdots d_{j}^{\alpha_{i^{\prime}-1}}+d_{j}^{\alpha_{i^{\prime}+1}} \cdots d_{j}^{\alpha_{|\Delta|}}\right)}{\sum_{l \neq i^{\prime}} d^{\alpha_{l}}} \leq 4 \kappa .
$$

By the inductive hypothesis,

$$
\sum_{l \neq i^{\prime}} d^{\alpha_{l}}\left(\pi_{\alpha_{l}}\left(\zeta\left(i_{0}\right)\right), \pi_{\alpha_{l}}\left(\zeta\left(i_{n}\right)\right)\right)=\sum_{l \neq i^{\prime}} d^{\alpha_{l}} \leq 2^{2(|\Delta|-2)} \hat{C}(4 \kappa) s .
$$

Finally, since $c_{\alpha_{i^{\prime}}} \leq c_{\beta_{i^{\prime}}}$, Lemma 3.1.4 says $d^{\alpha_{i^{\prime}}} \leq \sum_{l \neq i^{\prime}} d^{\alpha_{l}}$ which means

$$
d\left(\zeta\left(i_{0}\right), \zeta\left(i_{n}\right)\right)=d^{\alpha_{i^{\prime}}}+\sum l \neq i^{\prime} d^{\alpha_{l}} \leq 22^{2(|\Delta|-2)} \hat{C}(4 \kappa) s=2^{2(|\Delta|-1)} \hat{C}(2 \kappa) s .
$$

\subsection{Efficient scale}

This section is based on [6, Definition 4.5 and Lemma 4.6].

3.2.1 Definition ( $\epsilon$-Efficient at scale $\tilde{r}$ ) Let $Y$ be a metric space, and $\lambda:[0, L] \rightarrow Y$ a rectifiable curve. We say that $\lambda$ is $\epsilon$-efficient at scale $\tilde{r}, 0<\tilde{r} \leq 1$ if

$$
\sum_{j} d\left(p_{j}, p_{j+1}\right) \leq(1+\epsilon) d(\lambda(0), \lambda(L)), \quad \text { where }\left\{p_{j}\right\}=\hat{\mathcal{S}}(\lambda, \widetilde{r} d(\lambda(0), \lambda(L))) .
$$

3.2.2 Remark Note that being efficient at scale $r$ does not necessarily imply being efficient at all scales $\tilde{r}<r$.

Efficiency provides with us the closest description of being "straight" in $\mathbb{R}^{n}$, whose meaning is made precise by the following lemma.

3.2.3 Lemma If $\lambda:[a, b] \rightarrow \mathbb{R}^{n}$ is $\epsilon$-efficient at scale $r$, then

$$
d_{H}(\lambda, \overline{\lambda(a) \lambda(b)}) \leq\left(1.5 \epsilon^{1 / 4}+r\right) d(\lambda(a), \lambda(b)) .
$$

That is, $\lambda$ is close to a straight line. 
Proof Let $m=r d(\lambda(a), \lambda(b))$, and $\left\{p_{j}\right\}_{j=0}^{N}=\mathcal{S}(\lambda, m)$ so that $d\left(p_{0}, p_{N}\right)=$ $d(\lambda(a), \lambda(b))=L$. Let $h_{\overline{p_{0} p_{N}}}$ be the orthogonal projection of $\lambda$ onto $\overline{p_{0} p_{N}}$ and $\tilde{p}_{i}=h_{\overline{p q}}\left(p_{i}\right)$, so $d\left(\tilde{p}_{j}, \tilde{p}_{j+1}\right) \leq d\left(p_{j}, p_{j+1}\right)=m$. Since $\tilde{p}_{0}=p_{0}, \tilde{p}_{N}=p_{N}$, we have $\bigcup_{i=0}^{N-1} \widetilde{\tilde{p}_{i} \tilde{p}_{i+1}}=\overline{p_{0} p_{N}}$, and the Lemma in the Appendix gives that $d\left(p_{j}, \tilde{p}_{j}\right) \leq$ $1.5 \epsilon^{1 / 4} L$. So if $\dot{p} \in \lambda$, letting $p_{j}$ be the closest point in $\mathcal{S}(\lambda, m)$, we then have $d\left(\dot{p}, \overline{p_{0} p_{N}}\right) \leq d\left(\dot{p}, p_{j}\right)+d\left(p_{j}, \overline{p_{0} p_{N}}\right) \leq m+1.5 \epsilon^{1 / 4} L$. Similarly for $\ddot{p} \in \overline{p_{0} p_{N}}$, there is a $j$ such that $\ddot{p} \in \widetilde{\tilde{p}}_{j} \widetilde{p}_{j+1}$, with $d\left(\ddot{p}, \widetilde{p}_{j}\right) \leq d\left(\ddot{p}, \widetilde{p}_{j+1}\right)$ which gives $d(\ddot{p}, \lambda) \leq$ $d\left(\ddot{p}, \tilde{p}_{j}\right)+d\left(\tilde{p}_{j}, \lambda\right) \leq \frac{1}{2} m+1.5 \epsilon^{1 / 4} L$.

\subsection{Monotone scale}

The purpose of this section is to prove Proposition 3.3.2. It is based on [7, Section 4.1].

3.3.1 Definition ( $\delta$-Monotone) Let $G$ be a split abelian-by-abelian group, and $\zeta:[0, L] \longrightarrow G$, a $(\kappa, C)$ quasi-geodesic segment such that there exists a line segment $\overline{A B} \subset \mathbf{A}$ satisfying

$$
d_{H}\left(\pi_{A}(\zeta), \overline{A B}\right) \leq \epsilon\left\|\pi_{A}(\zeta)\right\|,
$$

for some $0 \leq \epsilon<1$. Let $h_{\overline{A B}}: \pi_{A}(\zeta) \rightarrow \overline{A B}$ be the map that sends every point of $\pi_{A}(\zeta)$ to the closest point on $\overline{A B}$ by orthogonal projection. We say that $\zeta$ is

- $\delta$-monotone, if whenever

$$
h_{\overline{A B}}\left(\pi_{A} \circ \zeta\left(t_{1}\right)\right)=h_{\overline{A B}}\left(\pi_{A} \circ \zeta\left(t_{2}\right)\right)
$$

we have

$$
d\left(\zeta\left(t_{1}\right), \zeta\left(t_{2}\right)\right) \leq \delta d(\zeta(0), \zeta(L))
$$

with $\epsilon\left|\pi_{A}(\zeta)\right| \leq \delta d(\zeta(0), \zeta(L))$.

- $\left(v, C_{1}\right)$ weakly monotone if whenever

$$
h_{\overline{A B}}\left(\pi_{A} \circ \zeta\left(t_{1}\right)\right)=h_{\overline{A B}}\left(\pi_{A} \circ \zeta\left(t_{2}\right)\right)
$$

for $t_{1}>t_{2}$, we have

$$
d\left(\zeta\left(t_{1}\right), \zeta\left(t_{2}\right)\right) \leq v d\left(\zeta\left(t_{1}\right), \zeta(0)\right)+C_{1},
$$

with $\epsilon\left|\pi_{A}(\zeta)\right| \leq C_{1} \leq v d\left(\zeta\left(t_{1}\right), \zeta(0)\right)$.

Note that the definition of weakly monotone is not symmetrical to both end points: it is biased towards the starting point $\zeta(0)$. The merit of a monotone path is that in a nondegenerate group, it is close to a geodesic segment as stated in the next proposition. 
3.3.2 Proposition Let $G$ be a nondegenerate, split abelian-by-abelian group, and $\lambda:[0, L] \rightarrow G$ a $(\kappa, C)$ quasi-geodesic whose $\pi_{A}$ image is $\epsilon$-efficient. Then

(i) when $\lambda$ is $\delta$ monotone, $\lambda$ is within $O(\delta L)$ neighborhood of a geodesic segment;

(ii) when $\lambda$ is $\left(\eta, C_{1}\right)$ weakly monotone, $\lambda$ is in $|\triangle| \eta$-linear $+O(1)$ (Recall that $\triangle$ is the set of roots of $G$ ) neighborhood of a geodesic.

Note that a monotone path is efficient by definition. So being close to a geodesic segment is the same as asking that the movement of the path along $\mathbf{H}$ direction (this is $\mathbf{H}$ in $G=\mathbf{A} \ltimes \mathbf{H}$ ) is not too big. We will prove Proposition 3.3.2 by using the observation that for a monotone path in $G$, admitting a geodesic approximation is the same as saying that for any root $\alpha \in \triangle$, its $\pi_{\alpha}$ (recall that $\pi_{\alpha}: G \longrightarrow \mathbb{H}_{\alpha}$; see last bullet point under "Subspaces of $G$ " in Section 2.2.4) image admits a (vertical) geodesic approximations (See Lemma 3.3.5 below).

The next lemma sets out one scenario where we have (vertical) geodesic approximation in $H_{n+1}=\mathbb{R}^{n} \rtimes_{\psi} \mathbb{R}$ Recall that $\psi(t)$ is $e^{t} N(t)$, where $N(t)$ is a nilpotent matrix with polynomial entries. We coordinatize points in $H_{n+1}$ as $(x, t)$, where $x \in \mathbb{R}^{n}$, and $t \in \mathbb{R}$. Let $h$ denotes the projection $(x, t) \mapsto t$.

3.3.3 Lemma Let $\left\{p_{i}\right\}_{i=-s}^{t}$, where $s, t \in \mathbb{Z}^{+}$, be points in $H_{n+1}$ such that for some $h_{0}>2, h\left(p_{j}\right)=h\left(p_{j-1}\right)+h_{0}$ for all $j$. For $i>0$, let $d_{i}$ denote the distance between $p_{i}$ and the vertical geodesic passing through $p_{i-1}$; for $i<0$, let $d_{i}$ denote for the distance between $p_{i}$ and the vertical geodesic passing $p_{i+1}$.

(i) If for all $j, d_{j} \leq r$, and $2 r \ll h_{0}$, then there is a geodesic $\gamma_{0}$ such that $d\left(\gamma_{0}, p_{j}\right) \leq 2 r$ for all $j$.

(ii) If for all $j, d_{j} \leq \eta|j|+C_{1}$, where $\eta \ll 1$ and $2 C_{1} \leq h_{0}$, then there is a geodesic $\gamma_{0}$ such that $d\left(\gamma_{0}, p_{j}\right) \leq 2 \eta|j|+2 C_{1}$.

Proof We first produce geodesic $\gamma^{+}$and $\gamma^{-}$that stay close to $\left\{p_{i}, i \geq 0\right\}$ and $\left\{p_{i}, i \leq 0\right\}$ respectively. Then we show that $\gamma^{+}$and $\gamma^{-}$coarsely intersect at some $p_{j}$, $j \geq 0$, and show that there is a geodesic $\gamma_{0}$ with the desired property.

Write $p_{j}=\left(x_{j}, t_{j}\right)$. We can assume without the loss of generality that $p_{0}=(0,0)$. Note that the distance between $\left(x_{1}, t_{1}\right)$ and the vertical geodesic passing through $\left(x_{2}, t_{2}\right)$ is $U\left(\left|x_{1}-x_{2}\right|\right)-t_{1}$ by Equation (3).

- Then, by Equation (4), for $j>0$,

$$
\ln \left|x_{j}-x_{j-1}\right|-j h_{0} \leq d_{j} .
$$


Hence for all $k \geq 0$,

$$
\left|x_{k}\right| \leq \sum_{j=1}^{k}\left|x_{j}-x_{j-1}\right| \leq \sum_{j} e^{d_{j}+j h_{0}}
$$

Let $\gamma^{+}$be the geodesic passing through $p_{0}$. Then for $k \geq 0$,

$$
d\left(p_{k}, \gamma^{+}\right) \leq 2 \ln \left(\sum_{j}^{k} e^{d_{j}+j h_{0}}\right)-2 k h_{0}=2 \ln \left(\sum_{j=1}^{k} e^{d_{j}+(j-k) h_{0}}\right)
$$

- For $j<0$, again by Equation (4)

hence

$$
\ln \left|x_{j+1}-x_{j}\right|-j h_{0} \leq d_{j},
$$

Note that under the assumptions of (i) or (ii), $x_{-\infty}=\lim _{j \rightarrow-\infty} x_{j}$ exists. So for all $k<0$,

$$
\left|x_{k}-x_{-\infty}\right| \leq \sum_{j=k-1}^{\infty} e^{d_{j}+j h_{0}}
$$

Let $\gamma^{-}$be the vertical geodesic passing through $\left(x_{-\infty}, 0\right)$. Then for $k<0$,

$$
d\left(p_{k}, \gamma^{-}\right) \leq 2 \ln \left(\sum_{k-1}^{-\infty} e^{d_{j}+j h_{0}}\right)-2 k h_{0}=2 \ln \left(\sum_{j=k-1}^{-\infty} e^{d_{j}+(j-k) h_{0}}\right) \text {. }
$$

(i) In this case, $d\left(p_{k}, \gamma^{+}\right) \leq 2 r$ for all $k \geq 0$; $d\left(p_{k^{\prime}}, \gamma^{-}\right) \leq 2 r$ for all $k^{\prime} \leq 0$. In particular, $d\left(p_{0}, \gamma^{-}\right) \leq 2 r$. Since $\gamma^{+} \ni p_{0}$, the height at which $\gamma^{+}$and $\gamma^{-}$ come together is at most $h\left(p_{0}\right)+2 r<h\left(p_{1}\right)$ by assumption. In other words, the parts of $\gamma^{-}$and $\gamma^{+}$above height $h\left(p_{0}\right)+2 r<h\left(p_{1}\right)$ are within $O(1)$ Hausdorff neighborhood of each other. So we can take $\gamma_{0}$ to be $\gamma^{-}$.

(ii) In this case, $d\left(p_{k}, \gamma^{+}\right) \leq(2 \eta) k+2 C_{1}$ for $k \geq 0$; $d\left(p_{k}, \gamma^{-}\right) \leq(2 \eta)(-k)+2 C_{1}$ for $k \leq 0$. In particular, $d\left(p_{0}, \gamma^{-}\right) \leq 2 C_{1}$, so the height at which $\gamma^{+}$and $\gamma^{-}$ come together occurs no higher than $h\left(p_{0}\right)+2 C_{1}$. For the same reason as in case (i), we can take $\gamma_{0}$ to be $\gamma^{-}$.

Recall that for each root $\alpha$, there is a corresponding projection map $\pi_{\alpha}: G \longrightarrow \mathbb{H}_{\alpha}$ onto a negatively curved space, and $h_{\alpha}: \mathbb{H}_{\alpha} \longrightarrow \mathbb{R}$ is the associated height projection.

3.3.4 Lemma Let $\gamma$ be a quasi-geodesic in $G$ such that for some weight $\alpha$, the image $h_{\alpha} \circ \pi_{\alpha}(\gamma)$ lies in a ball of radius $c_{2}$, where $c_{2} \ll\|\gamma\|$. Then $\pi_{\alpha}(\gamma)$ is within $O(\log (\|\gamma\|))$ Hausdorff neighborhood of a (vertical) geodesic segment. 
Proof Let $c$ be the additive constant of the quasi-isometry that defines $\gamma$. Then every time we move $C$ units in the domain of $\gamma, h_{\alpha} \circ \pi_{\alpha}(\gamma)$ moves at most $2 \kappa C$ units horizontally in $\mathbb{H}_{\alpha}$, but this means that by the end of the $\gamma, \pi_{\alpha}(\gamma(0))$ and $\pi_{\alpha}(\gamma(\|\gamma\|))$ is at most $O(\log (\|\gamma\|))$ apart.

3.3.5 Lemma Let $\gamma$ be a path in $G$ such that for each root $\alpha, \pi_{\alpha}(\gamma)$ is within $c_{1}$-Hausdorff neighborhood of a (vertical) geodesic, then $\gamma$ itself is within $O\left(c_{1}\right)$ Hausdorff neighborhood of (straight) geodesic.

Proof In order to specify a geodesic segment in $G$, we need to specify a direction in the $\mathbf{A}$ factor and a point in $\mathbf{H}$ factor. The latter is given by the base points of geodesics in each $\pi_{\alpha}(G)$. The former exists because the various geodesic segment approximate the $\pi_{\alpha}$ images of a fixed path.

We now proceed to prove Proposition 3.3.2 by showing that if a path is monotone, then for any root $\alpha$, its $\pi_{\alpha}$ image satisfies the hypothesis of Lemmas 3.3.3 and 3.3.4.

\section{Proof of Proposition 3.3.2 Set}

- $s=\delta\|\overline{A B}\|$

- $t_{j}=\max \left\{t \mid h_{\overline{A B}} \circ \pi_{A} \circ \lambda(t)=j s\right\}$

- $t_{j}^{\prime}=\min \left\{t \in\left[t_{j-1}, t_{j}\right] \mid h_{\overline{A B}} \circ \pi_{A} \circ \lambda(t)=j s\right\}$.

Therefore for $t \in\left[t_{j-1}, t_{j}^{\prime}\right]$, we must have $h_{\overline{A B}} \circ \pi_{A} \circ \lambda(t) \in[(j-1) s, j s]$. Since $d\left(\pi_{A}(\lambda), \overline{A B}\right) \leq \epsilon|\overline{A B}|$, the set $\left\{\pi_{A}(\lambda(t)), t \in\left[t_{j-1}, t_{j}^{\prime}\right]\right\}$ lies in a ball of diameter at most $\tilde{s}=\sqrt{s^{2}+(2 \epsilon|A B|)^{2}}=\sqrt{\delta^{2}+4 \epsilon^{2}}|A B|$, which means $d\left(\lambda\left(t_{j-1}\right), \lambda\left(t_{j}^{\prime}\right)\right) \leq \hbar \widetilde{s}$ by Proposition 3.1.1.

(i) In the case that $\lambda$ is $\delta$ monotone,

$$
h_{\overline{A B}}\left(\pi_{A} \circ \lambda\left(t_{j}\right)\right)=h_{\overline{A B}}\left(\pi_{A} \circ \lambda\left(t_{j}^{\prime}\right)\right) \Longrightarrow d\left(\lambda\left(t_{j}\right), \lambda\left(t_{j}^{\prime}\right)\right) \leq \delta d(\zeta(0), \zeta(L)) .
$$

(ii) If $\lambda$ is $\left(\eta, C_{1}\right)$ weakly monotone,

$$
h_{\overline{A B}}\left(\pi_{A} \circ \lambda\left(t_{j}\right)\right)=h_{\overline{A B}}\left(\pi_{A} \circ \lambda\left(t_{j}^{\prime}\right)\right) \Longrightarrow d\left(\zeta\left(t_{j}\right), \zeta\left(t_{j}^{\prime}\right)\right) \leq \eta d\left(\zeta\left(t_{j}\right), \zeta(0)\right)+C_{1} .
$$

Therefore $d\left(\lambda\left(t_{j-1}\right), \lambda\left(t_{j}\right)\right) \leq d\left(\lambda\left(t_{j-1}\right), \lambda\left(t_{j}^{\prime}\right)\right)+d\left(\lambda\left(t_{j}^{\prime}\right), \lambda\left(t_{j}\right)\right) \leq \Upsilon$, where

$$
\Upsilon= \begin{cases}\hbar \widetilde{s}+\delta d(\zeta(0), \zeta(L)) & \text { when } \lambda \text { is } \delta \text {-monotone, } \\ \hbar \widetilde{s}+\eta d\left(\zeta\left(t_{j}\right), \zeta(0)\right)+C_{1} & \text { when } \lambda \text { is }\left(\eta, C_{1}\right) \text { weakly monotone }\end{cases}
$$

The claim now follows from applying Lemmas 3.3.3 or 3.3.4 to $\left\{\pi_{\Xi}\left(\lambda\left(t_{j}\right)\right)\right\}_{j}$ in the $\Xi$ weight hyperbolic space for each root $\Xi$, followed by Lemma 3.3.5. 


\subsection{Finding a good scale}

In this section we show that given any $\epsilon, \delta$, if a quasi-geodesic is sufficiently long, then there is a scale at which the $\pi_{A}$ projection is $\epsilon$ efficient and the geodesic itself at that scale is $\delta$ monotone.

3.4.1 Proposition Let $G$ be a nondegenerate, split abelian-by-abelian group. Given any $N \gg 2,0<\epsilon, \delta, \eta<1$, satisfying $\delta / \hbar>1.5 \epsilon^{1 / 2}+\eta$, there is a number $L_{0}$ such that whenever $\zeta:[0, L] \rightarrow G$ is a $(\kappa, C)$ quasi-geodesic satisfying

$$
L_{0} \leq 2 \kappa L,
$$

then there is a scale $0<\rho_{J}<1$ such that

$$
\frac{\left|\mathcal{S}\left(S, \rho_{J}\|\zeta\|, \mathbf{P}\right)\right|}{\left|\mathcal{S}\left(\zeta, \rho_{J}\|\zeta\|\right)\right|} \leq \frac{1}{N}
$$

where $\mathbf{P}$ denotes the property "not within $\delta \rho_{J}\|\zeta\|$ Hausdorff neighborhoods of geodesic segments".

Proof Actually we will prove the statement

$$
\frac{\left|\mathcal{S}\left(S, \rho_{J}\|\zeta\|, \mathbf{Q}\right)\right|}{\left|\mathcal{S}\left(\zeta, \rho_{J}\|\zeta\|\right)\right|} \leq \frac{1}{N}
$$

for the statement $\mathbf{Q}$ being "not $\epsilon$ efficient or $\delta$ monotone", and the claim would then follow from Proposition 3.3.2.

If $\pi_{A}(\zeta)$ is already $\epsilon$-efficient and $\zeta$ itself $\delta$ monotone then we are done. Otherwise let $\rho_{i}=\eta^{i}, i=1,2, \ldots, D$ be $D$ many scales, and demand the curve be long enough so that it remains coarsely visible at the smallest scale. More precisely, demand $L_{0}$ to satisfy

$$
L_{0} \rho_{D} \geq 2 \kappa C
$$

We start the proof by setting

$$
\left\{p_{j}^{i}\right\}=\widehat{S}\left(\pi_{A}(\zeta), L_{i}\right), \quad \text { where } L_{i}=\rho_{i}\left\|\pi_{A}(\zeta)\right\|
$$

and let $\widetilde{p}_{j}^{i}$ be the corresponding point on $\zeta$ whose $\pi_{A}$ image is $p_{j}^{i}$. There are three possibilities for each $\zeta_{\left[\tilde{p}_{j}^{i}, \tilde{p}_{j+1}^{i}\right]}$ :

- $\pi_{A}(\zeta)_{\left[p_{j}^{i}, p_{j+1}^{i}\right]}$ is not $\epsilon$-efficient at scale $\eta$.

Say $p_{j}^{i}=p_{s_{1}}^{i+1}, p_{j+1}^{i}=p_{s_{2}}^{i+1}$. Then

$$
\sum_{t=s_{1}}^{s_{2}} d\left(p_{t}^{i+1}, p_{t+1}^{i+1}\right) \geq d\left(p_{j}^{i}, p_{j+1}^{i}\right)+\epsilon d\left(p_{j}^{i}, p_{j+1}^{i}\right)=d\left(p_{j}^{i}, p_{j+1}^{i}\right)+\epsilon L_{i}
$$


- $\pi_{A}(\zeta)_{\left[p_{j}^{i}, p_{j+1}^{i}\right]}$ is $\epsilon$-efficient at scale $\eta$ but $\zeta_{\left[\tilde{p}_{j}^{i}, \widetilde{p}_{j+1}^{i}\right]}$ is not $\delta$-monotone.

In this case there are two points $t_{1}, t_{2} \in\left[\zeta^{-1}\left(\tilde{p}_{j}^{i}\right), \zeta^{-1}\left(\tilde{p}_{j+1}^{i}\right)\right]$ such that

$$
h \overline{p_{j}^{i}, p_{j+1}^{i}}\left(\pi_{A} \circ \zeta\left(t_{1}\right)\right)=h \overline{\pi_{A}\left(p_{j}^{i}\right), \pi_{A}\left(p_{j+1}^{i}\right)}\left(\pi_{A} \circ \zeta\left(t_{2}\right)\right)
$$

with

$$
d\left(\zeta\left(t_{1}\right), \zeta\left(t_{2}\right)\right) \geq \delta d\left(\tilde{p}_{j}^{i}, \tilde{p}_{j+1}^{i}\right)
$$

Equation (9) and the assumption that $\zeta_{\left[p_{j}^{i}, p_{j+1}^{i}\right]}$ is $\epsilon$-efficient at scale $\eta$ imply that

$$
d\left(\pi_{A}\left(\zeta\left(t_{1}\right)\right), \pi_{A}\left(\zeta\left(t_{2}\right)\right)\right) \leq\left(1.5 \epsilon^{1 / 2}+\eta\right) L_{i}
$$

by Lemma 3.2.3.

On the other hand, by Corollary 3.1.2, (10) implies there must be a $t \in\left[t_{1}, t_{2}\right]$ such that

$$
d\left(\pi_{A}(\zeta(t)), \pi_{A}\left(\zeta\left(t_{1}\right)\right)\right) \geq \frac{\delta}{\hbar} L_{i}
$$

Say $p_{j}^{i}=p_{s_{1}}^{i+1}, P_{j+1}^{i}=p_{s_{2}}^{i+1}$. Then (11) and (12) imply that

$$
\begin{aligned}
\sum_{t=s_{1}}^{s_{2}-1} d( & \left.p_{t}^{i+1}, p_{t+1}^{i+1}\right) \\
& \geq d\left(p_{j}^{i}, \pi_{A}\left(\zeta\left(t_{1}\right)\right)\right)+d\left(\pi_{A}\left(\zeta\left(t_{2}\right)\right), p_{j+1}^{i}\right)+\sum_{\iota=1}^{2} d\left(\pi_{A}\left(\zeta\left(t_{\iota}\right)\right), \pi_{A}(\zeta(t))\right) \\
& \geq d\left(p_{j}^{i}, \pi_{A}\left(\zeta\left(t_{1}\right)\right)\right)+d\left(\pi_{A}\left(\zeta\left(t_{2}\right)\right), p_{j+1}^{i}\right)+\frac{2 \delta}{\hbar} L_{i}-d\left(\pi_{A}\left(\zeta\left(t_{1}\right)\right), \pi_{A}\left(\zeta\left(t_{2}\right)\right)\right) \\
& \geq d\left(p_{j}^{i}, p_{j+1}^{i}\right)+\frac{2 \delta}{\hbar} L_{i}-2 d\left(\pi_{A}\left(\zeta\left(t_{1}\right)\right), \pi_{A}\left(\zeta\left(t_{2}\right)\right)\right) \\
& \geq d\left(p_{j}^{i}, p_{j+1}^{i}\right)+2\left(\frac{\delta}{\hbar}-1.5 \epsilon^{1 / 2}-\eta\right) L_{i} .
\end{aligned}
$$

The first three lines are triangle inequalities, and the last one uses (11).

- In the remaining possibility we certainly have

$$
\sum_{t=s_{1}}^{s_{2}-1} d\left(p_{t}^{i+1}, p_{t+1}^{i+1}\right) \geq d\left(p_{j}^{i}, p_{j+1}^{i}\right) .
$$


Adding up (8), (13) and (14) we have

$$
\begin{aligned}
\sum_{j} d\left(p_{j}^{i+1}, p_{j+1}^{i+1}\right) \geq & \sum_{j} d\left(p_{j}^{i}, p_{j+1}^{i}\right)+\left(\begin{array}{c}
\text { number of } \\
\text { nonefficient segments }
\end{array}\right) \cdot \epsilon L_{i} \\
& +\left(\begin{array}{c}
\text { number of } \\
\text { nonmonotone segments }
\end{array}\right) \cdot \underbrace{2\left(\frac{\delta}{\hbar}-1.5 \epsilon^{1 / 2}-\eta\right)}_{\hat{\eta}} L_{i} \\
\geq & \sum_{j} d\left(p_{j}^{i}, p_{j+1}^{i}\right)+b_{i} \frac{\left\|\pi_{A}(\zeta)\right\|}{L_{i}} \epsilon L_{i}+\natural_{i} \frac{\left\|\pi_{A}(\zeta)\right\|}{L_{i}} \hat{\eta} L_{i} \\
= & \sum_{j} d\left(p_{j}^{i}, p_{j+1}^{i}\right)+\min \{\epsilon, \hat{\eta}\}\left(b_{i}+\natural_{i}\right)\left\|\pi_{A}(\zeta)\right\|,
\end{aligned}
$$

where $b_{i}$ and $\bigsqcup_{i}$ represent respectively, the proportion of nonefficient and nonmonotone segments. Writing $\lambda_{i}=\sum_{1 \leq j \leq n_{i}} d\left(p_{j}^{i}, p_{j+1}^{i}\right)$ and using (15) we have

$$
\left\|\pi_{A}(\zeta)\right\| \geq \lambda_{D}-\lambda_{0}=\sum_{i=0}^{D-1} \lambda_{i+1}-\lambda_{i} \geq \min \{\epsilon, \hat{\eta}\} \sum_{i=0}^{D-1}\left(b_{i}+\natural_{i}\right)\left\|\pi_{A}(\zeta)\right\| .
$$

Now divide both sides by $\left\|\pi_{A}(\zeta)\right\|$ to get

$$
\frac{1}{\min \{\epsilon, \hat{\eta}\}} \geq \sum_{i=0}^{D-1}\left(b_{i}+\natural_{i}\right)
$$

and note that by choosing $L_{0}$ large enough, we can take $D$ as large as needed, so there is a scale $\rho_{J}$ such that $b_{J}+\natural_{J} \leq 1 / N$.

3.4.2 Corollary Let $G$ be a nondegenerate, split abelian-by-abelian group. For any $2 \ll N_{0}<N, 0<\epsilon, \delta, \eta<1$, satisfying $\delta / \hbar>1.5 \epsilon^{1 / 2}+\eta$, there is a number $L_{0}$ such that whenever $\mathcal{F}=\left\{\zeta_{i}\right\}$ is a finite set of $(\kappa, C)$ quasi-geodesics such that every element of $\mathcal{F}, \zeta_{i}:\left[0, L_{i}\right] \rightarrow G$ satisfies

$$
L_{0} \leq 2 \kappa L_{i},
$$

then there is a scale $0<\rho_{J}<1$ and a subset $\mathcal{F}_{0}$ such that

(i) $\left|\mathcal{F}_{0}\right| \geq\left(1-N_{0} / N\right)|\mathcal{F}|$;

(ii) for every $\zeta_{i} \in \mathcal{F}_{0}$,

$$
\frac{\left|\mathcal{S}\left(\zeta_{i}, \rho_{J}\left\|\zeta_{i}\right\|, \mathbf{P}\right)\right|}{\left|\mathcal{S}\left(\zeta_{i}, \rho_{J}\left\|\zeta_{i}\right\|\right)\right|} \leq \frac{1}{N_{0}}
$$

where $\mathbf{P}$ is the statement of "not within $\delta \rho_{J}\|\zeta\|$ Hausdorff neighborhoods of a geodesic segment". 
Proof Apply Proposition 3.4.1 to each $\zeta_{i} \in \mathcal{F}$, and follow its proof until (16). Summing over all elements of $\mathcal{F}$ produces

$$
\frac{1}{\min \{\epsilon, \hat{\eta}\}} \geq \frac{1}{|\mathcal{F}|} \sum_{\zeta \in \mathcal{F}} \sum_{i=0}^{D-1}\left(b_{i}+\bigsqcup_{i}\right)=\sum_{i=0}^{D-1} \frac{1}{|\mathcal{F}|} \sum_{\zeta \in \mathcal{F}}\left(b_{i}+\bigsqcup_{i}\right) .
$$

The choice of $D$ implies there is a $1 \leq J<D$ such that

$$
\frac{1}{N} \geq \frac{1}{|\mathcal{F}|} \sum_{\zeta \in \mathcal{F}}\left(b_{i}+\natural_{i}\right) \text {. }
$$

Let $\mathcal{F}_{b}$ be those $\zeta \in \mathcal{F}$ whose $\left(b_{i}+\bigsqcup_{i}\right)$ value is more than $1 / N_{0}$. Applying Chebyshev we see that

$$
\frac{1}{N} \geq \frac{\left|\mathcal{F}_{b}\right|}{|\mathcal{F}|} \frac{1}{N_{0}}
$$

So the claim follows by taking $\mathcal{F}_{0}=\mathcal{F}-\mathcal{F}_{b}$.

\subsection{Proving Theorem 3.5.1}

Recall that we need to prove the following.

3.5.1 Theorem Let $G, G^{\prime}$ be nondegenerate, unimodular, split abelian-by-abelian Lie groups, and $\phi: G \rightarrow G^{\prime}$ be a $(\kappa, C)$ quasi-isometry. Given $0<\theta, \delta<1$, there are numbers $L_{0}, 0<\rho<1$ with the following properties.

If $\Omega \subset \mathbf{A}$ is a product of intervals of equal size at least $L_{0}$, then a tiling of $\mathbf{B}(\Omega)$ by isometric copies of $\mathbf{B}(\varrho \Omega)$

$$
\mathbf{B}(\Omega)=\bigsqcup_{j \in \mathbf{J}} \mathbf{B}\left(\omega_{j}\right) \sqcup \Upsilon
$$

contains a subset $\mathbf{J}_{0} \subset \mathbf{J}$ with a relative measure at least $1-\theta$ such that for all $j \in \mathbf{J}_{0}$,

(i) there is a subset $\mathcal{L}_{0}\left(\omega_{j}\right) \subset \mathcal{L}\left(\omega_{j}\right)$ with relative measure least $1-\theta$, such that their images under $\phi$ are within $\delta$ linear neighborhood of geodesic segments;

(ii) there is a subset $\mathcal{P}_{0}\left(\omega_{j}\right) \subset \mathcal{P}\left(\omega_{j}\right)$ with relative measure at least $1-\theta$, such that for every $p \in \mathcal{P}_{0}\left(\omega_{j}\right)$, at least $1-\theta$ proportion of geodesics passing within $O\left(\delta \operatorname{diam}\left(\omega_{j}\right)\right)$ of $x$, belong to $\mathcal{L}_{0}\left(\omega_{j}\right)$.

So far our results from previous sections only require the group to be nondegenerate and split abelian-by-abelian. From now on, we will require all our groups to be unimodular. 
3.5.2 Lemma Let $G$ be a nondegenerate, unimodular, split abelian-by-abelian Lie group. Let $\Omega \subset \mathbf{A}$ be a product of intervals of size $L_{0}$ centered at the origin, then for any $\rho=1 / n$, where $n \in \mathbb{N}$, we can write

$$
\mathbf{B}(\Omega)=\bigsqcup_{j} \mathbf{B}\left(\omega_{j}\right) \sqcup \Upsilon
$$

where the measure of the set $\Upsilon$ is at most $O(\rho)$ proportion of that of $\mathbf{B}(\Omega)$, and each $\mathbf{B}\left(\omega_{j}\right)$ is isometric to $\mathbf{B}(\rho \Omega)$.

Proof The unimodularity of $G$ guarantees that most of the volume is concentrated in the interior of $\mathbf{B}$. The tiling is just translates of the smaller box.

Proof of Theorem 3.5.1 Set $\epsilon=\delta^{4}, \eta=\delta^{8}, N=\lfloor 1 / \theta\rfloor, N_{0}=\sqrt{N}$ and apply Corollary 3.4.2 to $\phi(\mathcal{L}(\Omega))$ to obtain a scale $\rho_{J}$, a cut-off number $L_{0}$, and a large subset $\mathcal{F}_{0}$ of $\phi(\mathcal{L}(\Omega))=\mathcal{F}$.

Let $n=\left\lfloor 1 / \rho_{J}\right\rfloor$ and set $\rho=1 / n$. Then apply Lemma 3.5.2 to get a tiling of $\mathbf{B}(\Omega)$. Let $Y$ be the set consisting of pairs $(\zeta, x)$ where $\zeta \in \phi(\mathcal{L}(\Omega)), x \in \zeta$ and define $Y(x) \subset \mathcal{F}$ be those that pass within $\delta \rho \operatorname{diam}(\Omega)$ of $x$. Note that $|Y(x)|=c$ for some number $c$ depending on $\Omega$, so

$$
|Y|=\sum_{\zeta \in \mathcal{F}} \sum_{x \in \zeta} 1=\sum_{x \in \phi(\mathcal{P}(\Omega))} \sum_{\zeta \in Y(x)} 1=\sum_{x \in \phi(\mathcal{P}(\Omega))}|Y(x)|=c|\mathbf{B}(\Omega)| .
$$

If $f(\zeta, x)$ is a function on the set $Y$, we have

$$
\begin{aligned}
\frac{1}{|Y|} \sum_{\zeta \in \mathcal{F}} \sum_{x \in \zeta} f(\zeta, x) & =\frac{1}{|Y|} \sum_{x \in \phi(\mathcal{P}(\Omega))} \sum_{\zeta \in Y(x)} f(\zeta, x) \\
& =\frac{1}{|Y|} \sum_{x \in \phi(\mathcal{P}(\Omega))} \frac{1}{|Y(x)|} \sum_{\zeta \in Y(x)}|Y(x)| f(\zeta, x) \\
& =\frac{1}{c|\mathbf{B}(\Omega)|} \sum_{x \in \phi(\mathcal{P}(\Omega))} \frac{1}{|Y(x)|} \sum_{\zeta \in Y(x)} c f(\zeta, x) .
\end{aligned}
$$

Now apply (17) with $f$ being the characteristic function of the set $(\zeta, x)$ where $x \in \zeta$ and $\zeta \notin \mathcal{F}_{0}$. We get

$$
\frac{1}{|\mathbf{B}(\Omega)|} \sum_{x \in \phi(\mathcal{P}(\Omega))}\left(\frac{1}{|Y(x)|} \sum_{\zeta \in Y(x)} f(\zeta, x)\right)=O\left(1 / N_{0}\right) .
$$

The theorem now follows from Chebyshev applied to the function enclosed by the big parenthesis. 


\section{Inside of a box}

The purpose of this section is to give the proof of the main theorem of the paper.

Theorem 1.3.3 Let $G, G^{\prime}$ be nondegenerate, unimodular, split abelian-by-abelian Lie groups, and $\phi: G \rightarrow G^{\prime}$ be a $(\kappa, C)$ quasi-isometry. Given $0<\theta, \delta<1$, there are numbers $L_{0}, 0<\rho<1$ with the following properties.

If $\Omega \subset \mathbf{A}$ is a product of intervals of equal size at least $L_{0}$, then a tiling of $\mathbf{B}(\Omega)$ by isometric copies of $\mathbf{B}(\rho \Omega)$

$$
\mathbf{B}(\Omega)=\bigsqcup_{j \in \mathbf{J}} \mathbf{B}\left(\omega_{j}\right) \sqcup \Upsilon
$$

contains a subset $\mathbf{J}_{0} \subset \mathbf{J}$ of relative measure at least $1-\theta$, such that for all $j \in \mathbf{J}_{0}$, there is a subset $\mathcal{P}_{0}\left(\omega_{j}\right) \subset \mathcal{P}\left(\omega_{j}\right)$ with relative measure at least $1-\theta$ such that $\left.\phi\right|_{\mathcal{P}_{0}\left(\omega_{j}\right)}$ is within $O\left(\delta \operatorname{diam}\left(\omega_{j}\right)\right)$ of a standard map $g_{j} \times f_{j}$.

\subsection{Geometry of flats}

In this section, we note down some geometric properties of nondegenerate, unimodular, split abelian-by-abelian groups relevant to Theorem 1.3.3. Specifically, we will show if a large proportion of geodesics in a box are taken to bounded neighborhoods of geodesics under a quasi-isometry $\phi$, then most flats in that box are also taken to bounded neighborhoods of flats, and the restriction of $\phi$ to a large proportion of that box is close to a standard map.

4.1.1 Definition Given subsets $U_{i}$ 's in $G$, we say that they coarsely lie on a common flat if there is a flat $F$ and subsets $\widehat{U}_{i} \subset F$ such that the $d_{\mathcal{H}}\left(U_{i}, \widehat{U}_{i}\right)=O(1)$.

4.1.2 Lemma Let $G$ be a nondegenerate, split abelian-by-abelian group, and $\gamma, \zeta$ are geodesic segments in $G$ making an angle of at least $\sin ^{-1}(\widetilde{\eta})$ with root kernels such that for some $\eta \ll \tilde{\eta}<1$, and $\sup _{x \in \zeta} \inf _{y \in \gamma} d(x, y)=\eta(\|\gamma\|+\|\zeta\|)$. Then, $\gamma$ and $\zeta$ coarsely lie on a common flat for at least $\eta / \widetilde{\eta}$ proportion of their lengths.

Proof The assumption that $\gamma, \zeta$ make an angle at least $\sin ^{-1}(\widetilde{\eta})$ with the root kernels means that $\left\|\pi_{\alpha}(\gamma)\right\| \geq \tilde{\eta}\|\gamma\|,\left\|\pi_{\alpha}(\zeta)\right\| \geq \tilde{\eta}\|\zeta\|$. Now, if the claim is not true, then there is a root $\alpha$ such that $\pi_{\alpha}(\gamma)$ and $\pi_{\alpha}(\zeta)$ disagrees for more than $\eta / \widetilde{\eta}$ of their length. But this means that

$\sup _{x \in \zeta} \inf _{y \in \gamma} d(x, y) \geq \sup _{x \in \pi_{\alpha}(\zeta)} \inf _{y \in \pi_{\alpha}(\gamma)} d(x, y)>\frac{\eta}{\widetilde{\eta}}\left(\left\|\pi_{\alpha}(\gamma)\right\|+\left\|\pi_{\alpha}(\zeta)\right\|\right) \geq \eta(\|\gamma\|+\|\zeta\|)$, contradicting the assumption that $\sup _{x \in \zeta} \inf _{y \in \gamma} d(x, y)=\eta(\|\gamma\|+\|\zeta\|)$. 
4.1.3 Definition Let $G$ be a nondegenerate, split abelian-by-abelian Lie group. We define the following objects in $G$.

- A 2-simplex $\Delta$ is a set of three pair-wise intersecting geodesic segments. This includes the degenerate case of a geodesic segment and two subsegments of it. Elements of $\Delta$ are called edges of $\Delta$.

Note that a 2 -simplex is just a triangle. The term " 2 -simplex" is used here only because it makes the description more consistent.

- A filled 2-simplex $\widetilde{\Delta}$ consists of a 2 -simplex $\Delta$ together with all the 2simplices $\delta$ satisfying the following properties: two of the three edges of $\delta$ are subsegments of two edges of $\Delta$. The edges of $\Delta$ are called faces or edges of $\widetilde{\Delta}$.

For $I \geq 3$, we define

- an $I$-simplex $\Delta$ as a set of $I+1$ many filled $(I-1)$-simplices such that they pairwise intersect at their $I-2$ faces. An element of $\Delta$ is called an $(I-1)-$ face of $\Delta$.

- a filled $I$-simplex $\widetilde{\Delta}$ consists of an $i$-simplex $\Delta$ together with all $i$-simplices $\delta$ satisfying the following properties: $I$ many faces of $\delta$ are subsets of $I$ many faces of $\Delta$. By faces of $\widetilde{\Delta}$ we mean faces of $\Delta$. (See Figure 1.)

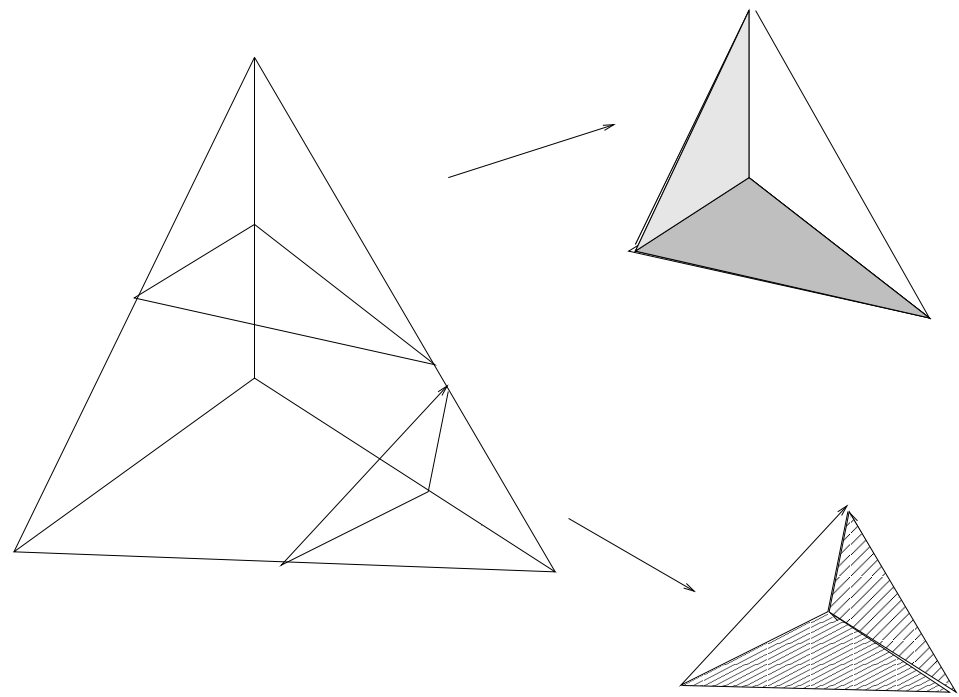

Figure 1: The big tetrahedron together with the two smaller shaded tetrahedron that are parts of a filled 3-simplex 
If the faces of a simplex behaves well under the quasi-isometry $\phi$, eg if their images under $\phi$ admit approximations by hyperplanes of appropriate dimensions, then we can approximate the image of a simplex by a simplex. This is the content of the next lemma, which deals with one instance where it is possible to approximate a quasi-simplex by a simplex.

4.1.4 Lemma Let $G$ be a nondegenerate, split abelian-by-abelian Lie group and $\mathcal{B}$ a family of geodesic segments such that

(i) $\max \{|\zeta|, 1 /|\zeta|, \zeta \in \mathcal{B}\}=M \ll \infty$;

(ii) for $\zeta \in \mathcal{B}, \phi(\zeta)$ is within $\eta|\phi(\zeta)|$ Hausdorff neighborhood of another geodesic segment $\widehat{\zeta}$, and $\widehat{\zeta}$ makes an angle at least $\sin ^{-1}(\widetilde{\eta})$ with the root kernels. We call $\hat{\zeta}$ a geodesic approximation of $\phi(\zeta)$.

Then for $I \leq n$, the $\phi$ images of any $I$-simplex or filled $I$-simplex made out of elements of $\mathcal{B}$ is within $O(\eta M)$ Hausdorff neighborhood of another simplex or filled simplex of the same dimension lying on a flat.

Proof We prove the claims by induction on $I$, starting with a $2-$ simplex, then filled 2 -simplex followed by 3 -simplex, filled 3 -simplex, etc....

The base step is the 2-simplex case. Before we start, we make the following observations. First, in $\mathbb{R}^{n}$, an $I$-simplex is entirely determined by its projection onto $n$ many 1 -dimension spaces given by a basis. Second, the image under $\pi_{\Xi}$ of an 2-simplex, whose edges are not parallel to any root kernels, is a collection of three pairwise intersecting vertical geodesic segments, ie a degenerate triangle on a line.

Now take a 2-simplex with sides labeled as $c_{1}, c_{2}$, and $c_{3}$. By assumption, their images under $\phi$ admits approximations by geodesic segments $\tilde{c}_{1}, \tilde{c}_{2}$ and $\tilde{c}_{3}$, with the properties that the end point of $\tilde{c}_{i}$ is no more than $\eta\left(\left\|c_{i}\right\|+\left\|c_{i+1}\right\|\right)$ away from the starting point of $\tilde{c}_{i+1}$. For each weight $\Xi$, each of $\pi_{\Xi}\left(\widetilde{c}_{i}\right)$ 's is a vertical geodesic segment. There are six possible configurations shown in Figure 2 below. To specify a 2-simplex on a flat that is close to these three geodesics, it is enough to specify the root space coordinates of this flat, and this is given by the root space coordinate of the gray line in each configuration.

To get an actual 2-simplex that approximates $\widetilde{c}_{1}, \widetilde{c}_{2}$ and $\widetilde{c}_{3}$ we can take it to be the 2 -simplex $\check{\Delta}$ that projects to the 2 -simplex approximations for at least a basis worth of weight vectors.

Now we examine the case of a filled 2 -simplex. Let $\widetilde{\Delta}=\{\Delta\} \cup\left\{\delta_{i}\right\}_{i}$ be a filled 2-simplex, and $\widehat{\Delta}, \widehat{\delta}_{i}$ 's denote for the 2-simplex approximation of $\phi(\Delta), \phi\left(\delta_{i}\right)$ 's, as 


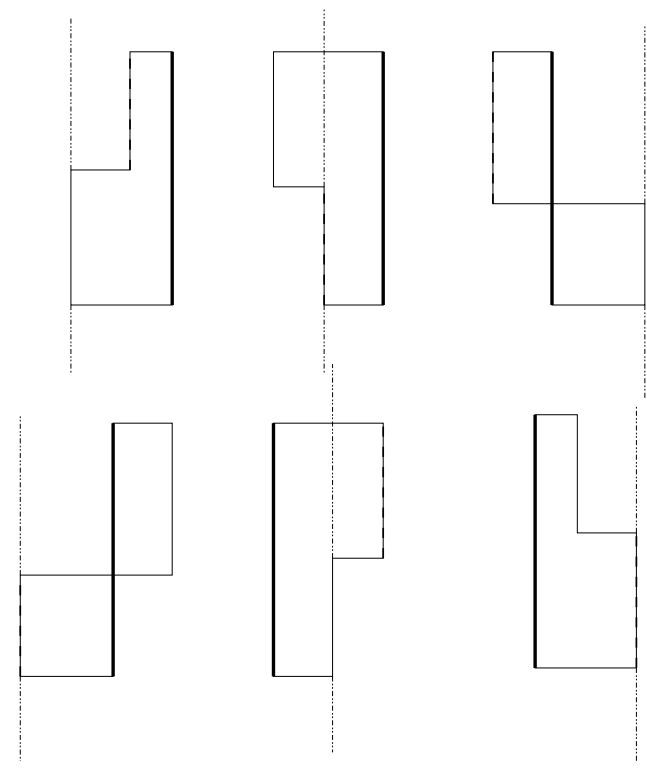

Figure 2: The six configurations in the Base step, 2-simplex case of proof to Lemma 4.1.4. The three vertical segments are $\tilde{c}_{i}, i=1,2,3$, and the gray line contains a degenerate 2 -simplex, whose faces are close to the $\widetilde{c}_{i}$ 's.

given by the 2-simplex case above. Fix a $i$. Let $\mathcal{F}$ and $\mathcal{F}_{i}$ represent the flats that house $\widehat{\Delta}$ and $\hat{\delta}_{i}$. By assumption, two edges $\gamma_{1}, \gamma_{2}$ of $\delta_{i}$ are subsegments of $\zeta_{1}$ and $\zeta_{2}$ of $\Delta$. Therefore there are $\check{\gamma}_{1}, \check{\gamma}_{2} \subset \hat{\delta}_{i}$ that approximate $\phi\left(\gamma_{1}\right)$ and $\phi\left(\gamma_{2}\right)$. Similarly, there are $\check{\zeta}_{1}$ and $\check{\zeta}_{2}$ in $\widehat{\Delta}$ that approximate $\phi\left(\zeta_{1}\right)$ and $\phi\left(\zeta_{2}\right)$. Therefore the pair $\left(\check{\gamma}_{i}, \check{\zeta}_{i}\right)$, satisfies the hypothesis of Lemma 4.1.2, and so $\mathcal{F}$ and $\mathcal{F}_{i}$ must have nontrivial coarse intersection (because the conclusion of Lemma 4.1.2 says that $\left(\tilde{\gamma}_{i}, \check{\gamma}_{i}\right)$ coarsely lie on a common flat). Since the coarse intersection between two flats is convex, therefore that there is a 2-simplex $\breve{\delta}_{i}$ lying on $\mathcal{F}$ such that $d_{H}\left(\widehat{\delta}_{i}, \breve{\delta}_{i}\right) \leq \eta M$, and $\widetilde{\gamma}_{1}, \tilde{\gamma}_{2} \subset \breve{\delta}_{i}$.

We now proceed with the inductive step and supposing that the claim is true for $i-$ simplices and their filled versions for $i \leq I-1$. Let $\Delta=\left\{\tilde{\delta}_{i}\right\}_{i=0}^{I}$ where each $\tilde{\delta}_{i}$ is a filled $(I-1)-$ simplex, and $\check{\delta}_{i}$ be their filled $I-1$ simplex approximations as yielded by the inductive hypothesis. Then we know for each weight $\Xi, \pi_{\Xi}\left(\check{\delta}_{i}\right)$ is a vertical geodesic segment, and for any $\check{\delta}_{i}, \check{\delta}_{j}, \pi_{\Xi}\left(\check{\delta}_{i}\right), \pi_{\Xi}\left(\check{\delta}_{j}\right)$ come together at some subsegment. If modulo $\eta / \widetilde{\eta}$ proportion of the ends, $\pi_{\Xi}(\check{\delta})$ 's do not coarsely lie on a common vertical geodesic segment, then the relationship between $\pi_{\Xi}\left(\check{\delta}_{i}\right), \pi_{\Xi}\left(\check{\delta}_{j}\right)$ is that of a forking $Y$; see Figure 3 below. But this contradicts the existence of another $\check{\delta}_{k}$ that shares a face with $\check{\delta}_{i}$ and another face with $\check{\delta}_{j}$. So modulo at most $\eta / \widetilde{\eta}$ proportion of their ends, $\pi_{\Xi}\left(\check{\delta}_{i}\right), \pi_{\Xi}\left(\check{\delta}_{j}\right)$ must coarsely lie on a common vertical geodesic. The 
same argument applied to every other weights means that we can translate each $\check{\delta}_{i}$ to $\breve{\delta}_{i}$ so that $\breve{\delta}_{i}, \breve{\delta}_{j}$ share a common face. The collection of all $\breve{\delta}_{i}$ 's forms our desired $\widehat{\Delta} I$-simplex. (Note that the inductive step is not a replacement of the 2 -simplex case in the base step because here, faces intersects at filled simplex of dimension $I-2$, which has diameter compatible to that of the diameter of the $I$-simplices of concern, whereas in the 2-simplex case, the pair-wise intersection of edges consist of just one point for each pair, so the same forking argument wouldn't work there.)

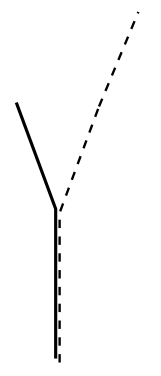

Figure 3: Inductive step, $I$-simplex case in the proof of Lemma 4.1.4: the solid and dotted lines represent $\pi_{\Xi}\left(\check{\delta}_{i}\right)$ and $\pi_{\Xi}\left(\check{\delta}_{j}\right)$.

We now examine the case of a filled $I$-simplex. Let $\widetilde{\Delta}=\{\Delta\} \cup\left\{\delta_{i}\right\}$ where each of $\Delta$ and $\delta_{i}$ is a $I$-simplex, and let $\widehat{\Delta}$ and $\hat{\delta}_{i}$ 's denote the $I$-simplex approximations of $\phi(\Delta)$ and $\phi\left(\delta_{i}\right)$ 's. Then for every $I$ many faces of $\hat{\delta}_{i}$ there are $I$ many corresponding faces of $\widehat{\Delta}$ to which they are a subset of, and this means the corresponding subsegments of edges of faces of $\widehat{\Delta}$ and the edges of faces of $\widehat{\delta}_{i}$ satisfy the hypothesis of Lemma 4.1.2, so they coarsely lie on a common flat. This means the flats housing $\widehat{\Delta}$ and $\widehat{\delta}_{i}$ respectively must coarsely intersect. Since the coarse intersection between two flats is convex, we conclude therefore that there is a $I$-simplex $\grave{\delta}_{i}$ in the flat containing $\widehat{\Delta}$ such that $d_{H}\left(\grave{\delta}_{i}, \hat{\delta}_{i}\right) \leq \eta M$, and $\grave{\delta}$ share $I$ of its faces with faces of $\widehat{\Delta}$. Then $\check{\Delta}=\{\widehat{\Delta}\} \cup\left\{\grave{\delta}_{i}\right\}$ has the desired property.

4.1.5 Definition Let $0 \leq \eta<1$. A $(\eta, C)$-quadrilateral $Q=\left\{\mathbf{T}_{i}\right\}_{i=0}^{3}$ in $G$ is a set of 4 oriented geodesic segments $\mathbf{T}_{i}$ satisfying the following.

(i) There is a nonsingular $\vec{v} \in \mathbf{A}$ such that the directions of $\mathbf{T}_{i}$ 's are all parallel to $\vec{v}$ (see Section 2.2.4 for definition of being singular).

(ii) $\left\|\mathbf{T}_{i}\right\|>2 \eta \sum_{j=0}^{3}\left\|\mathbf{T}_{j}\right\|$, for every $i$.

(iii) For all $i$ taken mod 4, some number $C \ll \eta \max \left\{\left\|\mathbf{T}_{i}\right\|\right\}$,

- $d\left(e_{i}, b_{i+1}\right) \leq \eta\left(\left\|\mathbf{T}_{i}\right\|+\left\|\mathbf{T}_{i+1}\right\|\right)+C$,

- $d\left(b_{i}, e_{i+1}\right) \geq(1-\eta)\left(\left\|\mathbf{T}_{i}\right\|+\left\|\mathbf{T}_{i+1}\right\|\right)+C$,

where $b_{i}, e_{i}$ denote the beginning and end points of $\mathbf{T}_{i}$. 
We refer to $\mathbf{T}_{i}$ 's as edges of $Q$, and write $\operatorname{diam}(Q)$ for the maximum length of its edges. The orientation of $\mathbf{T}_{i}$ is "up" (resp. "down") if its direction is parallel to a positive (resp. negative) multiple of $\vec{v}$.

Example Suppose the rank of $G$ is 1 . Let $V_{+}, V_{-}$denote for the two root class horocycles based at the identity element. Let $x \in V_{+}, y \in V_{-}$, and the word $x y x^{-1} y^{-1}$ represents a loop in $\mathbf{H}=V_{+} \oplus V_{-}$. If we choose $d(e, \tilde{x}), d(e, \tilde{y})=O(1)$, such that $x=t \tilde{x} t^{-1}$, and $y=t^{-1} \tilde{y} t$, then we obtain a loop representing a quadrilateral. Note that the same construction works if $G$ is rank 1 and nonunimodular, as long as there are expanding and contracting leaves.

4.1.6 Remark The first requirement of a quadrilateral means a quadrilateral exists in any nondegenerate rank 1 solvable abelian-by-abelian Lie group. Since any higher rank, nondegenerate, abelian-by-abelian solvable Lie group contains at least one rank 1 subgroup, quadrilaterals exist in higher rank for the same reason that they exist rank 1 spaces as illustrated by the previous example.

4.1.7 Lemma Let $Q=\left\{\mathbf{T}_{i}\right\}_{i=0}^{3}$ be a $(\eta, C)$-quadrilateral. Then the direction of $\mathbf{T}_{i}$ and $\mathbf{T}_{i+2}$ are positive multiple of each other, and that of $\mathbf{T}_{i}$ and $\mathbf{T}_{i+1}$ are negative multiple of each other.

Proof There are 16 possibilities to the relationship among directions of all the $\mathbf{T}_{i}$ 's (being positive or negative multiples of each other). One checks that only the combination stated above is allowed. An argument is given in the Appendix.

Let $A(t)$ be a 1-parameter matrix consisting of blocks of the form $e^{\alpha t} N(t)$ where $\alpha \neq 0, N(t)$ a nilpotent matrix with polynomial entries, and $\mathbb{R} \ltimes_{A} \mathbb{R}^{m}$ be a semidirect product for which $r \in \mathbb{R}$ acts on $\mathbb{R}^{m}$ by linear map $A(r)$. Write an element of $\mathbb{R} \ltimes_{A} \mathbb{R}^{m}$ as $(r, \mathbf{x})$, where $r \in \mathbb{R}, \mathbf{x} \in \mathbb{R}^{m}$, and $W^{+}$(resp. $W^{-}$) for the direct sum of positive (resp. negative ) eigenspaces of $A(t)$.

4.1.8 Lemma In $\mathbb{R} \ltimes_{A} \mathbb{R}^{m}$, suppose for some $\eta \ll 1$, we have $r_{0}, r_{1}, r_{2}, r_{3}>0$, $u_{0}, u_{2} \in W^{+}, u_{1}, u_{3} \in W^{-}$such that for index $j$ taken mod 4 satisfy

- $d\left(u_{j}, e\right) \leq \eta\left(r_{j}+r_{j+1}\right)$ for all $j$;

- $r_{j} \geq 2 \eta \sum_{\iota=0}^{3} r_{\iota}$ for all $j$;

- the word $\left(r_{0}, 0\right) u_{0}\left(-r_{1}, 0\right) u_{1}\left(r_{2}, 0\right) u_{2}\left(-r_{3}, 0\right) u_{3}$ is trivial.

Then $\left|r_{i}-r_{i+1}\right| \leq d\left(e, u_{i+1}\right)+d\left(e, u_{i+3}\right)$. In particular this implies that the sizes of the $r_{i}$ 's are equal up to an error of at most $\eta \sum_{i=0}^{3} r_{i}$.

Proof See the Appendix. 
4.1.9 Remark The main jest of the Lemma 4.1.8 is that the length of a quadrilateral is essentially determined by one number.

4.1.10 Lemma Let $Q=\left\{\mathbf{T}_{i}\right\}_{i=0}^{3}$ be a $(\eta, C)$-quadrilateral. Then

(i) $\left\|\mathbf{T}_{i}\right\|-\left\|\mathbf{T}_{j}\right\| \leq \eta\left(\sum_{i=0}^{3}\left\|\mathbf{T}_{i}\right\|\right)$ for all $i, j$;

(ii) $\left|\pi_{\vec{v}} \circ \Pi_{\vec{v}}\left(e_{i}\right)-\pi_{\vec{v}} \circ \Pi_{\vec{v}}\left(b_{i-1}\right)\right| \leq d\left(e_{i}, b_{i+1}\right)+d\left(e_{i+2}, b_{i+3}\right)$ for all $i$;

(iii) $\left\{\Pi_{\vec{v}}\left(b_{i}\right), \Pi_{\vec{v}}\left(e_{i+1}\right), \Pi_{\vec{v}}\left(b_{i+2}\right), \Pi_{\vec{v}}\left(e_{i+3}\right)\right\}$ are within $\eta\left(\sum_{i=0}^{3}\left\|\mathbf{T}_{i}\right\|\right)$ neighborhood of a coset of $W_{\vec{v}}^{+}$(or $W_{\vec{v}}^{-}$) if $i=0 \bmod 2$, and of a coset of $W_{\vec{v}}^{-}$(or $W_{\vec{v}}^{+}$) otherwise.

Proof Modifying $\mathbf{T}_{i}$ 's by an amount of at most $\eta \sum_{j}\left\|\mathbf{T}_{j}\right\|$, we can assume $\pi_{A}\left(e_{i}\right)=$ $\pi_{A}\left(b_{i+1}\right)$ for all $i$. Furthermore, the divergent assumption between $b_{i}$ and $e_{i+1}$ means that $e_{i}^{-1}\left(b_{i+1}\right) \in W_{\vec{v}}^{+}$(resp. $\left.W_{\vec{v}}^{-}\right)$if the direction of $\mathbf{T}_{i}$ is positive (resp. negative) multiples of $\vec{v}$. The result now follows from Lemma 4.1.8.

A schematic illustration for a quadrilateral with the correct orientation and lengths for its edges is given in Figure 4 below.

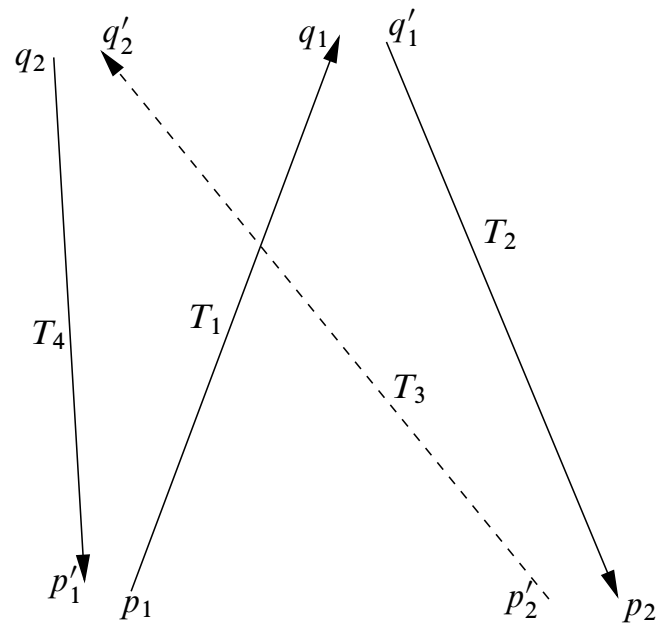

Figure 4: A schematic illustration of a quadrilateral

4.1.11 Lemma Let $Q=\left\{\gamma_{j}\right\}_{j=0}^{3}$ be a $(0, C)$-quadrilateral in $G$ such that each $\gamma_{j}$ is properly contained in a geodesic segment $\tilde{\gamma}_{j}$, whose $\phi$ image is within $\eta\left\|\tilde{\gamma}_{j}\right\|$ neighborhood of another geodesic segment whose direction is parallel to a nonsingular $\vec{v}_{j} \in \mathbf{A}$. Suppose further that $\left\|\tilde{\gamma}_{j} \cap \tilde{\gamma}_{j+1}\right\|>\eta \sum_{l=0}^{3}\left\|\tilde{\gamma}_{l}\right\|$. Then, there is an $\left(\hat{\eta}, C^{\prime}\right)-$ quadrilateral $\hat{Q}$ satisfying $d_{H}(\phi(Q), \widehat{Q}) \leq \hat{\eta} \operatorname{diam}(Q)$, where $\hat{\eta}=\max \left\{\eta\left(\left\|\tilde{\gamma}_{j}\right\| /\left\|\gamma_{j}\right\|\right)\right\}_{j}$ and $C^{\prime}=\kappa C+C$. 
Proof For each $j$, let $\widetilde{T}_{j}$ be an geodesic approximation of $\phi\left(\tilde{\gamma}_{j}\right)$. Since $Q$ is a $(0, C)$-quadrilateral, $\tilde{\gamma}_{j} \cap \tilde{\gamma}_{j+1}$ is a geodesic segment with positive length, the angle between the $\vec{v}_{j}$ and $\vec{v}_{j+1}$ is at most $\sin ^{-1}(\eta)$, and $d\left(\widetilde{T}_{j}, \widetilde{T}_{j+1}\right) \leq \eta\left(\left\|\widetilde{T}_{j}\right\|+\left\|\widetilde{T}_{j+1}\right\|\right)$. By moving each $\widetilde{T}_{j}$ by an amount at most $\eta \sum_{l}\left\|\widetilde{T}_{l}\right\|$, we can assume the directions of $\widetilde{T}_{j}$ 's are all parallel to some $\vec{v}$ with $W_{\vec{v}}^{0}=\{0\}$, and $\widetilde{T}_{j} \cap \widetilde{T}_{j+1}$ is a geodesic segment of positive length. Let $\mathbf{T}_{j} \subset \widetilde{T}_{j}$ be the subsegment closest to $\phi\left(\gamma_{j}\right)$. Then $\widehat{Q}=\left\{\mathbf{T}_{j}\right\}$ is a $\left(\hat{\eta}, C^{\prime}\right)$-quadrilateral.

4.1.12 Remark In practice, when we apply Lemma 4.1.11 and Remark 4.1.12 to the quasi-isometric image of a quadrilateral, we will often omit mentioning the extended edges $\tilde{\gamma}_{j}$ 's.

\subsection{Averaging}

In this section, we put together some of the observations in the last two sections to show that if a large percentage of geodesic segments in a box admit geodesic approximations to their $\phi$ images, then for $i \geq 2$, a large percentage of $i$-hyperplanes in the box also admit $i$-hyperplane approximations to their $\phi$ images. In particular, there is a large subset of flats in the box whose $\phi$ images are close flats.

The following is an averaging lemma that will be used repeatedly for the reminder of this section.

4.2.1 Lemma Let $\left(A, \mu_{\alpha}\right),\left(B, \mu_{\beta}\right)$ be two finite measure space, and $\sim$ is a relation between them. For $a \in A$, write $B_{a}=\{b \in B, b \sim a\}$ as the subset of $B$ consisted of elements related to $a$, and $A_{b}$, for $b \in B$, as the subset of elements of $A$ related to $b$.

Suppose $\mu_{\beta}\left(B_{a}\right) / \mu_{\beta}\left(B_{a^{\prime}}\right) \leq M_{A}$ for any $a, a^{\prime} \in A$, and $\mu_{\alpha}\left(A_{b}\right) / \mu_{\alpha}\left(A_{b^{\prime}}\right) \leq M_{B}$ for any $b, b^{\prime} \in B$.

If for some $s \leq(1) / M_{A} M_{B}, A^{s} \subset A$ with $\mu_{\alpha}\left(A^{s}\right) \leq s \mu_{\alpha}(A)$, then the subset $B^{s, t}=$ $\left\{b \in B: \mu_{\alpha}\left(A_{b} \cap A^{s}\right) \geq t \mu_{\alpha}\left(A_{b}\right)\right\}$, satisfies $\mu_{\beta}\left(B^{s, t}\right) \leq(s / t) M_{A} M_{B} \mu_{\beta}(B)$.

Proof See the Appendix.

4.2.2 Remark Lemma 4.2.1 will often be used to show that for subset $A^{0} \subset A$ of relative large measure, the subset of $B$ consisting of elements $b \in B$ such that the measure of $A_{b} \cap A^{0}$ is large relative to that of $A_{b}$, has large relative measure.

4.2.3 Lemma Let $G=\mathbf{H} \rtimes \mathbf{A}, G^{\prime}=\mathbf{H}^{\prime} \rtimes \mathbf{A}^{\prime}$ be nondegenerate, unimodular, split abelian-by-abelian Lie groups, and $\phi: G \rightarrow G^{\prime}$ be a $(\kappa, C)$ quasi-isometry. Let $\Omega \subset \mathbf{A}$ be a product of intervals of equal size. Suppose for some $0 \leq \delta<\theta<1$, there are subsets $\mathcal{L}^{0} \subset \mathcal{L}(\Omega), \mathcal{P}^{\prime} \subset \mathcal{P}(\Omega)$, both with relative measures at least $1-\theta$, with the following properties: 
- If $\zeta \in \mathcal{L}^{0}$, then $\phi(\zeta)$ admits a straight geodesic approximation.

- If $p \in \mathcal{P}^{\prime}$, then at least $1-\theta$ proportion of geodesics within $\delta \operatorname{diam}(\Omega)$, belongs to $\mathcal{L}^{\prime}$.

There is a subset $\mathcal{P}^{0} \subset \mathcal{P}^{\prime}$ of relative measure at least $1-\eta$ such that if $p, q \in \mathcal{P}^{0}$ are two points on the same flat, then $\phi(p), \phi(q)$ are within $O(\delta \operatorname{diam}(\Omega))$ neighborhood of a flat.

4.2.4 Remark The conclusion of Lemma 4.2.3 implies that a flat whose points are mostly in $\mathcal{P}^{\prime}$ is mapped close to another flat under $\phi$. This is because if not, then we would have three points $p, q, r$ such that the flat that is close to $\phi(p)$ and $\phi(q)$ is not within $O(\delta \operatorname{diam}(\Omega))$ neighborhoods of the flat close to $\phi(q)$ and $\phi(r)$. So then under some weight space projection, the configurations of the three points would trace out a fork as in Figure 3 in the proof of Lemma 4.1.4, contradicting the assumption that $\phi(p)$ and $\phi(r)$ are close to a common flat.

Proof The idea of the proof is to set up a situation where we can apply Lemma 4.1.4. We now construct subsets $\mathcal{L}_{i}^{0} \subset \mathcal{L}_{i}(\Omega)$ for $i=1,2,3, \ldots, n$, and $\mathcal{P}^{0} \subset \mathcal{P}(\Omega)$, all with relative proportion at least $1-\theta$ such that

- for every $S \in \mathcal{L}_{i}^{0}(\Omega)$,

$$
\left|\mathcal{L}_{i-1}(S) \cap \mathcal{L}_{i-1}^{0}\right| \geq(1-\theta)\left|\mathcal{L}_{i-1}(S)\right| ;
$$

- for every $p \in \mathcal{P}^{0}$,

$$
\left|L_{i}(p) \cap \mathcal{L}_{i}(\Omega) \cap \mathcal{L}_{i}^{0}\right| \geq(1-\theta)\left|L_{i}(p) \cap \mathcal{L}_{i}(\Omega)\right|,
$$

where $L_{i}(p)$ means the $i$-dimensional hyperplanes that contain $p$.

We construct those subsets inductively, starting with base case when $i=2$, since $\mathcal{L}_{1}^{0}=\mathcal{L}^{0}$ is already given.

Incidence is a relation between $\mathcal{L}(\Omega)$ and $\mathcal{L}_{2}(\Omega)$, so by Lemma 4.2.1 we can choose $s_{2}(\theta)<1$ appropriately such that the set

$$
\mathcal{L}_{2}^{0}=\left\{S \in \mathcal{L}_{2}(\Omega):\left|L_{1}(S) \cap \mathcal{L}(\Omega) \cap \mathcal{L}^{0}\right| \geq\left(1-s_{2}\right)\left|L_{1}(S) \cap \mathcal{L}(\Omega)\right|\right\}
$$

satisfies $\quad\left|\mathcal{L}_{2}^{0}\right| \geq\left(1-\theta^{1 / 2}\right)|\mathcal{L}(\Omega)|$.

Fix a $S \in \mathcal{L}_{2}^{0}$. Define $P(S)^{\text {bad }} \subset P(S)$ as

$$
P(S)^{\text {bad }}=\left\{p \in \mathcal{P}(\Omega):\left|L(p) \cap L(S) \cap \mathcal{L}(\Omega) \backslash \mathcal{L}^{0}\right| \geq s_{b}|L(p) \cap L(S) \cap \mathcal{L}(\Omega)|\right\} .
$$


We obtain a bound on the relative size of $P(S)^{\text {bad }}$ as follows.

Let $\chi$ be the characteristic function of the subset of

$$
\{(p, \zeta): p \in P(S), \zeta \in L(S) \cap \mathcal{L}(\Omega), p \in \zeta\}
$$

consisting of pairs $(p, \zeta)$ where $\zeta \in L(S) \cap\left(\mathcal{L}(\Omega) \backslash \mathcal{L}^{0}\right)$. Then, starting from

$$
\sum_{x \in P(S)} \sum_{\gamma \in L(x) \cap L(S) \cap \mathcal{L}(\Omega)} \chi=\sum_{\gamma \in L(S) \cap \mathcal{L}(\Omega)} \sum_{p \in P(\gamma)} \chi
$$

we have

$$
\begin{aligned}
& \sum_{x \in P(S)} \sum_{\gamma \in L(x) \cap L(S) \cap \mathcal{L}(\Omega)[m]} \chi \geq \sum_{x \in P(S)^{\mathrm{bad}}} s_{b}|L(x) \cap L(S) \cap \mathcal{L}(\Omega)[m]| \\
& \geq s_{b}\left|P(S)^{\mathrm{bad}}\right| \min \{|L(x) \cap L(S) \cap \mathcal{L}(\Omega)|: x \in S\}, \\
& \sum_{\gamma \in L(S) \cap \mathcal{L}(\Omega)} \sum_{p \in \gamma} \chi \leq s_{2}|L(S) \cap \mathcal{L}(\Omega)| \max \{|P(\zeta)|: \zeta \in L(S) \cap \mathcal{L}(\Omega)\}
\end{aligned}
$$

which yields

$$
\begin{aligned}
\left|P(S)^{\mathrm{bad}}\right| & \leq \frac{s_{2}}{s_{b}} \frac{|L(S) \cap \mathcal{L}(\Omega)| \max \{|P(\zeta)|: \zeta \in L(S) \cap \mathcal{L}(\Omega)\}}{\min \{|L(x) \cap L(S) \cap \mathcal{L}(\Omega)|: x \in S\}} \\
& \leq \frac{s_{2}}{s_{b}} k|P(S)|
\end{aligned}
$$

where $k$ depends only on $G$. By choosing $s_{b}=s_{2}^{1 / 2}$, we have that the measure of $P(S)^{\text {bad }}$ is at least $1-s_{2}^{1 / 2}$ times that of $P(S)$.

We now apply Lemma 4.2.1 to $P(S), L(S) \cap \mathcal{L}(\Omega)$, and $P(S)^{\text {bad }}$ to conclude that for some $v_{2} \ll 1$, the subset

$$
L(S)^{\mathrm{bad}}=\left\{\zeta \in L(S) \cap \mathcal{L}(\Omega):\left|P(\zeta) \cap P(S)^{\mathrm{bad}}\right| \geq \nu_{2}|P(\zeta)|\right\}
$$

satisfies

$$
\left|L(S)^{\mathrm{bad}}\right| \leq v_{2}^{\prime}|L(S)|
$$

for some $v_{2}^{\prime} \ll 1$ depending on $v_{2}$. Apply Lemma 4.2.1 again to $P(S), L(S) \cap \mathcal{L}(\Omega)$, and $L(S)^{\text {good }}=\left(L(S)-L(S)^{\text {bad }}\right) \cap \mathcal{L}^{0}$ to conclude that for some $\widehat{v}_{2} \ll 1$, the subset

$$
P(S)^{w}=\left\{p \in P(S):\left|L(p) \cap \mathcal{L}(\Omega) \cap L(S)^{\operatorname{good}}\right| \leq\left(1-\widehat{v}_{2}\right)|L(p) \cap \mathcal{L}(\Omega)|\right\}
$$

satisfies

$$
\left|P(S)^{w}\right| \leq \tilde{v}_{2}|P(S)|
$$

for some $\tilde{\nu}_{2} \ll 1$. Now set $P(S)^{0}$ as $P(S)-P(S)^{\text {bad }}-P(S)^{w}$, and let $\mathcal{P}_{2}$ as the union of $P(S)^{0}$ as $S$ ranges over $\mathcal{L}_{2}^{0}$. 
In general, once $\mathcal{L}_{i-1}^{0}$ is constructed, we repeat the argument above by replacing subscript 1 and 2 by $i-1$ and $i$ respectively, to arrive at subsets $\mathcal{L}_{i}^{0}, P(S)^{0} \subset P(S)$ for every $S \in \mathcal{L}_{i}^{0}$, and $\mathcal{P}_{i}$ as the union of all the $P(S)^{0}$ as $S$ varies over $\mathcal{L}_{i}^{0}$. Set $\mathcal{P}^{0}$ as the intersection of all the $\mathcal{P}_{i}$ 's. Since each $\mathcal{P}_{i}$ has large proportion relative to $\mathcal{P}(\Omega)$, the same is true for $\mathcal{P}^{0}$.

We now show that $\mathcal{P}^{0}$ has the claimed property. Take $p, q \in \mathcal{P}^{0}$. We claim that it sufficient to assume that $d(q, \partial \mathbf{B}(\Omega)) \geq \frac{1}{3} \operatorname{diam}(\mathbf{B}(\Omega))$, and $d(p, q) \geq \frac{1}{3} \operatorname{diam}(\mathbf{B}(\Omega))$. To see that this is sufficient observe that if the initial pair $p, q$ fails either conditions, we find a third point $z_{0} \in \mathcal{P}^{0}$ from the same flat such that $p, z_{0}$ and $q, z_{0}$ do, and that $\phi(p)$ and $\phi(q)$ lies within $O(\delta \operatorname{diam}(\Omega))$ neighborhood of a flat follows at once from triangle inequality applied to the pairs $\phi(p), \phi(z)$ and $\phi(q), \phi(z)$.

Denote the set of codimension 1 hyperplanes in $\mathbf{B}(\Omega)$ through $p$ and $q$ by $\mathrm{Gr}_{n-1}(p)$ and $\operatorname{Gr}_{n-1}(q)$ respectively. We place on them invariant measures coming from viewing them as homogeneous spaces. To each element of $\mathrm{Gr}_{n-1}(p)$ that does not contain $q$, we give it an orientation (that is, an element $\vec{n} \in \mathbf{A}$ ) so that it points to the half space containing $q$. Similarly for an element of $\mathrm{Gr}_{n-1}(q)$ that does not contain $p$, we give it a normal that points towards the half space containing $p$. We proceed to extract a nonempty subset $\mathrm{Gr}_{\text {good }} \subset\left(\mathrm{Gr}_{n-1}(p)\right)^{n} \times \mathrm{Gr}_{n-1}(q)$ such that its element gives us a $n$ simplex containing $p$ and $q$, by making the following demands. If $\left(Q_{1}, Q_{2}, \ldots, Q_{n}, S\right) \in \mathrm{Gr}_{\text {good }}$, then

(1) $p \in Q_{i}$, but $d\left(q, Q_{i}\right) \geq 2 \delta \operatorname{diam}(\mathbf{B}(\Omega))$ for all $i$, and $q \in S$ but $d(p, S) \geq$ $2 \delta \operatorname{diam}(\mathbf{B}(\Omega))$;

(2) $d\left(q, Q_{i} \cap S\right) \leq(1 / 6) \operatorname{diam}(\mathbf{B}(\Omega))$ for every $i$;

(3) $0<\angle \vec{n}_{Q_{i} \cap S}, \vec{n}_{Q_{j} \cap S} \leq 2 \cos ^{-1}((1 / 6) /(1 / 3-\delta))$, for every $i, j$, where $\vec{n}_{Q_{i} \cap S}$ denotes the normal of $Q_{i} \cap S$ in $S$ as inherited from $Q_{i}$, and $\angle \vec{n} Q_{i} \cap S, \vec{n} Q_{j} \cap S$ means the angles between them.

We now justify the existence of $\mathrm{Gr}_{\text {good }}$. First, we note that the subset of $\mathrm{Gr}_{n-1}(p)$ that comes within $2 \delta \operatorname{diam}(\mathbf{B}(\Omega))$ of $q$ has measure $O(\delta)$ relative to the whole of $\mathrm{Gr}_{n-1}(p)$. This is because in order for an element of $\mathrm{Gr}_{n-1}(p)$ to come within $2 \delta \operatorname{diam}(\mathbf{B}(\Omega))$ of $q$, the angle between its normal and the vector $\overrightarrow{p q}$ has to lie in the interval centered at $\pi / 2$ of width $O(\delta)$. The reasoning is symmetrical in $p$ and $q$, so we conclude that the subset of $\left(\mathrm{Gr}_{n-1}(p)\right)^{n} \times \mathrm{Gr}_{n-1}(q)$ that fails condition (1) has relative measure $O(\delta)$.

Now fix a $S \in \mathrm{Gr}_{n-1}(q)$ and consider the subset of $\mathrm{Gr}_{n-1}(p)$ satisfying condition (2). Certainly this is a nonempty set because we can just take a hyperplane $S^{\prime} \subset S$ of 
codimension 1 (in $S$ ) that is no more than $(1 / 6) \operatorname{diam}(\mathbf{B}(\Omega))$ away from $q$ and let $Q \in \mathrm{Gr}_{n-1}(p)$ be the element determining by $S^{\prime}$ and $p$. Conversely every element of $\mathrm{Gr}_{n-1}(p)$ satisfying condition (2) arises this way. Furthermore, if $Q \in \mathrm{Gr}_{n-1}(p)$ fails condition (2), then by writing out the linear equation for $Q$ using the distance between the $S^{\prime}=Q \cap S$ and $q$, we see that failing condition (2) is equivalent to saying that the angle between $\vec{n}_{S}$ and $\vec{n}_{Q}$ lies outside of some interval $I$, with size less than $\pi$ and depends only on the choice $1 / 6$ and the $1 / 3$ appearing in the lower bound of $d(p, q)$. (Note that we state an lower bound on the angle between $\vec{n}_{S}$ and $\vec{n}_{Q}$ here because our particular choices of orientations on the normals.) So in this way see that the subset of $\mathrm{Gr}_{n-1}(p)$ meeting the requirement of condition (2) is a set of positive measure.

Keeping the $S$ fixed as before, we now impose condition (3) on the set that satisfies condition (2). From the previous paragraph we identify $Q \in \mathrm{Gr}_{n-1}(p)$ satisfying condition (2) with a pair $(z, r)$, where $2 \delta \operatorname{diam}(\mathbf{B}(\Omega))<r<d(q, \partial \mathbf{B}(\Omega))-\delta \operatorname{diam}(\mathbf{B}(\Omega))$, and $z \in \mathbb{S}^{n-2}(q)$ is an element of the radius 1 sphere centered at $q$, such that the vector from $q$ to the point on $Q$ closest to it is $r \overrightarrow{q z}$. By the way, $\overrightarrow{q z}$ is parallel to $\vec{n}_{Q}$, the normal of $Q$, but with opposite orientation.

In doing so, we set up a diffeomorphism between the subset of $\mathrm{Gr}_{n-1}(p)$ satisfying condition (2) with the product measure between that of $\mathbb{S}^{n-2}$ and the interval $(2 \delta \operatorname{diam}(\mathbf{B}(\Omega)), d(q, \partial \mathbf{B}(\Omega))-\delta \operatorname{diam}(\mathbf{B}(\Omega)))$. We can thus identify those two sets and use the measures on either spaces, since they are related up to constant multiple. Now given $Q_{i}, Q_{j}$ satisfying condition (2), let $\left(z_{i}, r_{i}\right),\left(z_{j}, r_{j}\right)$ be the aforementioned pairs corresponding to them. By drawing a great circle through $z_{i}$ and $z_{j}$ and recalling that $d(q, \partial \mathbf{B}(\Omega)) \geq \frac{1}{3} \operatorname{diam}(\mathbf{B}(\Omega))$, we see at once that condition (3) is the same as asking the spherical distance $d\left(z_{i}, z_{j}\right)$ be nonzero and smaller than $2 \cos ^{-1}((1 / 6) /(1 / 3-\delta))$.

Putting the last paragraphs together we see that subset of $\mathrm{Gr}_{n-1}(p)^{n}$ satisfying (2) and (3) has positive measure, and the set $\mathrm{Gr}_{\text {good }}$ has positive measure also because the subset of $\mathrm{Gr}_{n-1}(p)^{n} \times \mathrm{Gr}_{n-1}(q)$ failing condition (1) goes to zero with $\delta$ while the relative measure of those fulfilling conditions (2) and (3) does not decrease with $\delta$.

Now we show elements of $\mathrm{Gr}_{\text {good }}$ give rise to simplices. Take $\left(Q_{1}, Q_{2}, \ldots, Q_{n}, S\right) \in$ $\mathrm{Gr}_{\text {good }}$. If we can show that their nonempty pairwise intersections (inside of $\mathbf{B}(\Omega)$ ) are of the right dimension then we are done. This is certainly true of the intersections between the $S$ and the $Q_{i}$ 's by virtue of condition (2). As for intersections between the $Q_{i}$ 's, demanding that their intersection inside of the box to have the correct codimension is the same as asking the intersection between $Q_{i} \cap S$ and $Q_{j} \cap S$ to have nonempty intersection with the interior of the box, since $Q_{i} \cap S$ and $Q_{j} \cap S$ are not parallel by assumption, and any two nonparallel codimension 1 hyperplanes intersect 
at a codimension 2 subspace. We can make sure their intersections intersect the interior of the box by demanding that the $d\left(q, Q_{i} \cap S \cap Q_{j}\right)<d(q, \partial \mathbf{B}(\Omega))-\delta \operatorname{diam}(\mathbf{B}(\Omega))$. By considering the quadrilateral with one vertex in $S \cap Q_{i} \cap Q_{j}$ and the remaining being $q$, and the points on $Q_{i} \cap S, Q_{j} \cap S$ closest to $q$ we have the statement of condition (3).

So now $\phi(p)$ and $\phi(q)$ lie within $O(\delta \operatorname{diam}(\Omega))$ of a common flat by Lemma 4.1.4.

\subsection{Proof of Theorem 1.3.3}

Apply Theorem 3.5.1 to $\mathbf{B}(\Omega)$ to obtain a tiling

$$
\mathbf{B}(\Omega)=\bigsqcup_{i \in \mathbf{I}} \mathbf{B}\left(\omega_{i}\right) \cup \Upsilon
$$

where each $\mathbf{B}\left(\omega_{i}\right)$ is isometric to $\mathbf{B}(\rho \Omega)$ and a subset $\mathbf{I}_{0} \subset \mathbf{I}$ of relative large measure such that for every $i \in \mathbf{I}_{0}$, there is a subset $\mathcal{L}_{i}^{\prime} \subset \mathcal{L}\left(\omega_{i}\right)$ of relative large measure whose images under $\phi$ are within $O\left(\delta \operatorname{diam}\left(\omega_{i}\right)\right)$ neighborhoods of geodesic segments. Fix one such $i \in \mathbf{I}_{0}$, and apply Lemma 4.2.3 and Remark 4.2.4 to conclude that $\left.\phi\right|_{\mathcal{P}^{\prime}}$ sends flats to flats.

To obtain a product structure on $\mathcal{P}^{0}$, we proceed to show that $\left.\phi\right|_{f}$ and $\left.\phi\right|_{f^{\prime}}$ for $f, f^{\prime} \in \mathcal{L}_{\operatorname{rank}(G)}^{0}$ are identical up to an error of $\eta \operatorname{diam}\left(\mathbf{B}\left(\Omega_{i}\right)\right)$. In the process of doing so, we will show that left cosets of root spaces are sent to left cosets of root spaces, and left cosets of $\mathbf{H}$ are sent to left cosets of $\mathbf{H}^{\prime}$ up to an error of the same order.

First we show that the claim is true for two flats $f, f^{\prime} \in \mathcal{L}_{\operatorname{rank}(G)}^{0}\left(\omega_{i}\right)$ that contain points $p \in f \cap \mathcal{P}^{0}\left(\omega_{i}\right), p^{\prime} \in f^{\prime} \cap \mathcal{P}^{0}\left(\omega_{i}\right)$ such that $p, p^{\prime}$ lie on a common root class horocycle, and $d\left(p, p^{\prime}\right) \geq 8 \delta \operatorname{diam}\left(\omega_{i}\right)$.

Since to any pairs of distinct root classes there exists at least one nonsingular vector such that the pair has nontrivial intersection with the corresponding expanding and contracting subspaces, it follows that a root class can be uniquely defined by the its signs (expanding or contracting) with respect to some $O(1)$ number of nonsingular directions in $\mathbf{A}$. So for $p, p^{\prime} \in \mathcal{P}^{0}\left(\omega_{i}\right) \subset \mathcal{P}^{0}$, we can find geodesic segments $l_{p, 1}, l_{p, 2} \in \mathcal{L}^{0}(\Omega)$ containing $p, l_{q, 1}, l_{q, 2} \in \mathcal{L}^{0}(\Omega)$ containing $q$ such that for some subsegments $\widehat{l}_{*, \iota} \subset l_{*, \iota}, *=p, q$, $\iota=1,2, Q=\left\{\widehat{l}_{p, \iota}, \widehat{l}_{q, \iota}\right\}_{\iota=1,2}$ is a $(0, C)$-quadrilateral.

As $d(p, q) \geq 8 \delta \operatorname{diam}\left(\omega_{i}\right)$, by Lemma 4.1 .11 and Remark 4.1.12, there is a $(O(\delta), \widehat{C})-$ quadrilateral $\hat{Q}$ within $O\left(\delta \operatorname{diam}\left(\omega_{i}\right)\right)$ Hausdorff distance away from $\phi(Q)$. Applying Lemma 4.1.10 to $\hat{Q}$, we see that $\phi(p)$ and $\phi\left(p^{\prime}\right)$ are within $O\left(\delta \operatorname{diam}\left(\omega_{i}\right)\right)$ neighborhood of a left translate of $W_{\vec{v}}^{+}$or $W_{\vec{v}}^{-}$where $\vec{v}$ is the direction of edges of $\widehat{Q}$. Since $p, q \in \mathcal{P}^{0}\left(\omega_{i}\right)$, we can build quadrilaterals $Q_{1}, Q_{2}, \ldots, Q_{k}$ for $k \leq n+2$, the 
edges of each are elements of $\mathcal{L}^{0}(\Omega)$ such that their respective approximating quadrilaterals $\hat{Q}_{1}, \hat{Q}_{2}, \ldots, \hat{Q}_{k}$, with edge directions $\vec{v}_{1}, \vec{v}_{2}, \ldots, \vec{v}_{k}$ satisfies $\bigcap_{\iota=1}^{k} W_{\vec{v}_{\iota}}^{\sigma(\iota)}$ with $\sigma(\iota) \in\{+,-\}$, is $V_{[\alpha]}$ for some root class $[\alpha]$. For the same reason as before, we see that $\phi(p)$ and $\phi(q)$ lie within $O\left(\delta \operatorname{diam}\left(\omega_{i}\right)\right)$ Hausdorff neighborhood of a translate $W^{\sigma(\iota)}$ for $\iota=1,2, \ldots, k$, therefore $\phi(p)$ and $\phi(q)$ lie within $O\left(\delta \operatorname{diam}\left(\omega_{i}\right)\right)$ Hausdorff neighborhood of a translate of $V_{[\alpha]}$. By using more quadrilaterals, the argument above also shows that $\left.\phi\right|_{f \cap \mathcal{P}^{0}\left(\omega_{i}\right)}$ are the same as $\left.\phi\right|_{f^{\prime} \cap \mathcal{P}^{0}\left(\omega_{i}\right)}$ up to an error of $O\left(\delta \operatorname{diam}\left(\omega_{i}\right)\right)$.

In general, for two arbitrary points $p, q \in \mathcal{P}_{i}^{0}$ in the same left coset of $\mathbf{H}$, we can find at most $2|\triangle|$ number of points $p_{0}=p, p_{1}, p_{2}, \ldots, p_{l}=q$, such that each consecutive pair of points lie on a common root class horocycle, and distance at least $8 \delta \operatorname{diam}\left(\omega_{i}\right)$ away. The quadrilateral argument above then shows that $\phi(p)$ are $\phi(q)$ within $O\left(\delta \operatorname{diam}\left(\omega_{i}\right)\right)$ neighborhood of a translate of $\mathbf{H}^{\prime}$.

Let $f: \mathbf{A} \rightarrow \mathbf{A}^{\prime}$ be a map that is within $O\left(\delta \operatorname{diam}\left(\omega_{i}\right)\right)$ away from $\left.\phi\right|_{f \cap \mathcal{P}^{0}\left(\omega_{i}\right)}$ for any flat $f \in \mathcal{L}_{n}^{0}\left(\omega_{i}\right)$. We now show that $f$ has to preserve root kernels. Take $p \in \mathcal{P}^{0}\left(\omega_{i}\right)$, and $f_{1}, f_{2}$ be two flats in $\mathcal{L}_{n}^{0}\left(\omega_{i}\right)$ containing $p$. Then $p \in f_{1} \cap f_{2}$, so $f_{1} \cap f_{2} \neq \varnothing$, hence $\phi\left(f_{1}\right) \cap \phi\left(f_{2}\right) \neq \varnothing$, and any two flat approximations to $\phi\left(f_{1}\right)$ and $\phi\left(f_{2}\right)$ have nonempty intersection as well. Since two flats come together at a convex set whose boundary is a union of hyperplanes parallel to root kernels, this means that the restriction of $\phi$ to any flats $f \in \mathcal{L}_{n}^{0}\left(\omega_{i}\right)$ preserves hyperplanes parallel to root kernels up to an error of $O\left(\delta \operatorname{diam}\left(\omega_{i}\right)\right)$.

\section{Appendix}

Proof of Lemma 3.1.3 We will use the notation from Equation (3). Write $p=(x, t)$, $q=\left(x^{\prime}, t^{\prime}\right)$. By assumption, $\left|t-t^{\prime}\right| \leq s$. If $U\left(\left|x-x^{\prime}\right|\right) \leq \min \left\{t, t^{\prime}\right\}$, then assume $t \geq t^{\prime}$. Then

$$
d\left((x, t),\left(x^{\prime}, t^{\prime}\right)\right) \leq d\left((x, t),\left(x^{\prime}, t\right)\right)+d\left(\left(x^{\prime}, t\right),\left(x^{\prime}, t^{\prime}\right)\right) \leq 2\left(t-t^{\prime}\right)+1 \leq 3 s
$$

and we are done. Now suppose $U\left|x-x^{\prime}\right| \geq t, t^{\prime}$, but $U\left|x-x^{\prime}\right| \leq 4 s$. Then

$$
\begin{aligned}
d\left((x, t),\left(x^{\prime}, t^{\prime}\right)\right) \leq & d\left((x, t),\left(x, U\left(\left|x-x^{\prime}\right|\right)\right)\right)+d\left(\left(x, U\left(\left|x-x^{\prime}\right|\right)\right),\left(x^{\prime}, U\left(\left|x-x^{\prime}\right|\right)\right)\right) \\
& +d\left(\left(x^{\prime}, U\left(\left|x-x^{\prime}\right|\right)\right),\left(x^{\prime}, t^{\prime}\right)\right) \leq 2 U\left(\left|x-x^{\prime}\right|\right)-\left(t+t^{\prime}\right)+1 \\
\leq & 8 s+1 \leq 12 \kappa s
\end{aligned}
$$

and we are done.

Finally suppose $U\left(\left|x-x^{\prime}\right|\right) \geq t, t^{\prime}$, and $U\left(\left|x-x^{\prime}\right|\right) \geq 4 s$. Since $\eta$ is continuous, we can find $i_{0} \leq i_{1} \leq i_{2} \leq i_{3} \leq \cdots \leq i_{n} \in[a, b]$ and therefore points $\left\{p_{j}=\eta\left(i_{j}\right)\right\}_{j=1}^{n}$ such 
that $p=(x, t)=\eta\left(i_{0}\right)=p_{0}, p_{n}=\eta\left(i_{n}\right)=q=\left(x^{\prime}, t^{\prime}\right)$, and $U\left(\left|x_{j}-x_{j+1}\right|\right)=4 s$, for all $j$ except maybe the last one, where $U\left(\left|x_{n-1}-x_{n}\right|\right) \leq 4 s$.

Then by Equation (3),

$$
\frac{\sum_{j=0}^{n-1}\left(U\left(\left|x_{j}-x_{j+1}\right|\right)-\left(t_{j}+t_{j+1}\right)\right)}{\left(U\left(\left|x_{0}-x_{n}\right|\right)-\left(t_{0}+t_{n}\right)\right)} \leq \frac{\sum_{j=0}^{n-1} d\left(p_{j}, p_{j+1}\right)}{d\left(p_{0}, p_{n}\right)} \leq 2 \kappa .
$$

Simplifying using Equation (4) yields

$$
\frac{(n-1) 2 s}{2 \ln \left(n e^{4 s}\right)} \leq 2 \kappa
$$

which means $(n-1) s \leq 2 \kappa(\ln (n)+4 s)$, so $n \leq 20 \kappa$. So

$$
d\left(p_{0}, q_{0}\right) \leq \sum_{j=0}^{n-1} d\left(p_{j}, p_{j+1}\right) \leq \sum_{j=0}^{n-1}\left(U\left(\left|x_{j}-x_{j+1}\right|\right)-\left(t_{j}+t_{j+1}\right)\right) \leq 20 \kappa s=80 \kappa s .
$$

Proof of Lemma 3.1.4 The claim is clear if $c_{\alpha}=1$. Otherwise we know

$$
\frac{c_{\alpha}}{c_{\beta}}=\frac{|b / B-(a+b) /(A+B)|}{|a / A-(a+b) /(A+B)|}
$$

Therefore $c_{\alpha} \geq c_{\beta}$ gives us that

$$
\left|\frac{a}{A}-\frac{a+b}{A+B}\right| \leq\left|\frac{b}{B}-\frac{a+b}{A+B}\right| .
$$

- Suppose $b / B<a / A$. Writing $b=c_{1} a, B=c_{2} A$, we have

$$
\begin{aligned}
1-\frac{1+c_{1}}{1+c_{2}} & <\frac{1+c_{1}}{1+c_{2}}-\frac{c_{1}}{c_{2}}, \\
c_{2}\left(c_{2}-1\right) & <c_{1}\left(c_{2}-1\right),
\end{aligned}
$$

If $A<B=c_{2} A$, then $1<c_{2}$, and this gives us $c_{2}<c_{1}$, which means $1<c_{1} / c_{2}$. Multiplying both sides by $a / A$ this means $a / A<b / B$, contradiction. So $A \geq B$.

- Now suppose $a / A<b / B$. Then again, that $a / A$ is closer to $(a+b) /(A+B)$ than $b / B$ means

$$
\begin{aligned}
\frac{a+b}{A+B}-\frac{a}{A} & <\frac{b}{B}-\frac{a+b}{A+B}, \\
\frac{1+c_{1}}{1+c_{2}}-1 & <\frac{c_{1}}{c_{2}}-\frac{1+c_{1}}{1+c_{2}}, \\
c_{1}\left(c_{2}-1\right) & <c_{2}\left(c_{2}-1\right) .
\end{aligned}
$$

If $A<B$, then $c_{2}>1$, and this gives us $c_{1}<c_{2}$, which means $c_{1} / c_{2}<1$. Multiplying by $a / A$ this says $b / B<a / A$, contradiction. So $A \geq B$. 
Lemma Given a triangle in $\mathbb{R}^{2}$ with vertices $A, B, C$ and opposites of length $a, b, c$, satisfying $(a+b) / c \leq 1+\epsilon$ for some $\epsilon \in[0,0.5]$, then

- $d(C, \overline{A B}) \leq 1.5 \epsilon^{1 / 4} \overline{A B}$;

- $\min \{A, B\} \leq \max \left\{\pi-\cos ^{-1}(-1+\sqrt{\epsilon /(1+\epsilon)}), \sin ^{-1}(\sqrt{\epsilon /(1+\epsilon)} / 2)\right\}$.

Proof The condition on the length means

$$
1 \geq \frac{c^{2}}{(a+b)^{2}}=\frac{(a+b)^{2}-2 a b(1+\cos (C))}{(a+b)^{2}} \geq \frac{1}{1+\epsilon} .
$$

Writing $1 /(1+\epsilon)=1-\hat{\epsilon}$, (note that $\hat{\epsilon}=1-1 /(1+\epsilon) \leq \epsilon)$ for some small $\hat{\epsilon}>0$, we have

$$
0 \leq \frac{2 a b}{(a+b)^{2}}(1+\cos (c)) \leq \hat{\epsilon}
$$

which means either $(1+\cos (C)) \leq \sqrt{\hat{\epsilon}}$ or $2 a b /(a+b)^{2} \leq \sqrt{\hat{\epsilon}}$.

In the first case, $\cos (C) \leq-(1-\sqrt{\hat{\epsilon}})$, so $A, B<A+B \leq \pi-\cos ^{-1}(-1+\sqrt{\hat{\epsilon}})$ and

$$
\begin{aligned}
d(C, \overline{A B})=|\overline{A C}| \sin (A) & \leq|\overline{A B}| \sin \left(\pi-\cos ^{-1}(-1+\sqrt{\hat{\epsilon}})\right) \\
& =|\overline{A B}| \sin \left(\cos ^{-1}(-1+\sqrt{\hat{\epsilon}})\right) .
\end{aligned}
$$

Hence $\quad d(C, \overline{A B}) \leq|\overline{A B}| \sqrt{1-(1-\sqrt{\hat{\epsilon}})^{2}} \leq|\overline{A B}| \sqrt{(1-1+\sqrt{\hat{\epsilon}})(1+1-\sqrt{\hat{\epsilon}})}$

$$
\leq|\overline{A B}| \sqrt{2 \sqrt{\hat{\epsilon}}} \text {. }
$$

In the second case, the condition is equivalent to

$$
\frac{2 \sin (A) \sin (B)}{(\sin (A)+\sin (B))^{2}} \leq \sqrt{\widehat{\epsilon}}
$$

Divide top and bottom by $\sin (B)$ (if $\sin (A)=\sin (B)=0$ then we are done, so assume one of them is not zero) so

$$
2 \sin (A) \leq 2 \frac{\sin (A)}{\sin (B)} \leq \frac{2(\sin (A) / \sin (B))}{(1+\sin (A) / \sin (B))^{2}} \leq \sqrt{\hat{\epsilon}}
$$

yields $A \leq \sin ^{-1}(\sqrt{\hat{\epsilon}} / 2)$. Since $\epsilon \leq 0.5, \hat{\epsilon}=1-(1) / 1+\epsilon \leq \frac{1}{3}$. So $\angle A \leq 16.78^{\circ}$. Since $C+B=\pi-A$, Without loss of generality $C \geq B, C \geq \pi-A /(2) \geq 45^{\circ}$ so 
$\tan (C) \geq 1$. Therefore

$$
\begin{aligned}
\frac{|\overline{A C}|}{|\overline{A B}|} & =\frac{\sin (B)}{\sin (C)}=\frac{\sin (\pi-C-A)}{\sin (C)}=\frac{\sin (\pi-C) \cos (A)}{\sin (C)}-\frac{\sin (A) \cos (\pi-C)}{\sin (C)} \\
& =\cos (A)+\frac{\sin (A)}{\tan (C)} \leq \cos (A)+\sin (A) \leq 2 .
\end{aligned}
$$

Hence $\quad d(C, \overline{A B})=\sin (A)|\overline{A C}| \leq \sin (A) 2|\overline{A B}| \leq \frac{\sqrt{\widehat{\epsilon}}}{2} 2|\overline{A B}|=\sqrt{\widehat{\epsilon}}|\overline{A B}|$.

Proof of Lemma 4.1.7 The quadrilateral is the same as the loop below.

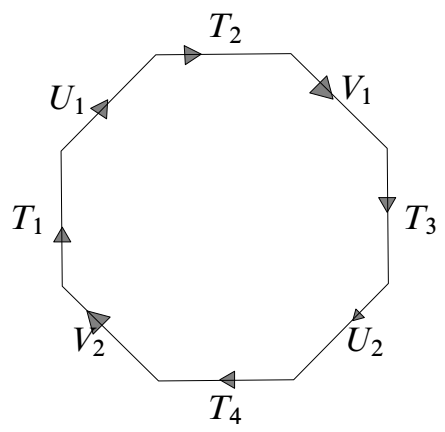

Figure 5: The loop given by a quadrilateral

Write $\mathbf{T}_{i}=T_{i} v$. Since $\left|U_{1}\right|,\left|U_{2}\right|,\left|V_{1}\right|,\left|V_{2}\right|$ are all less than $\eta\left(\sum\left|\mathbf{T}_{i}\right|\right)$, the first claim that $\sum_{i=1}^{4} T_{i} \leq \eta\left(\sum_{i=1}^{4}\left|\mathbf{T}_{i}\right|\right)$ follows by walking around the loop associated to $Q$.

So it cannot be the case that all the $T_{i}$ 's are of the same sign. Without loss of generality we can assume $T_{2}>0$, and $T_{3}<0$. Furthermore, regardless of the signs of the remaining $T_{i}$ 's, there must be another pair of adjacent $T_{i}$ 's of opposite signs, and either this pair involves one of $\left\{T_{2}, T_{3}\right\}$, or that it doesn't. In the latter case, $T_{1}>0$ and $T_{4}<0$, and the projection of this quadrilateral into $\langle v\rangle \ltimes \mathbb{R}^{m}$ is a quadrilateral with two consecutive upward and two consecutive downward edges, and such a quadrilaterals doesn't exist. So either $T_{2}$ or $T_{3}$ is involved in a pair of oppositely signed edges. Without loss of generality, we assume $T_{1}<0$. Then by (iv) in the definition of a quadrilateral, we have that $d\left(e, \Pi_{W_{v}^{+}}\left(U_{1}\right)\right) \geq 1$, because $T_{1}<0$ and $T_{2}>0$; and $d\left(e, \Pi_{W_{v}^{-}}\left(V_{1}\right)\right) \geq 1$, because $T_{2}>0$ and $T_{3}<0$, where $\Pi_{W_{v}^{+}}:(x, t) \mapsto \pi_{W_{v}^{+}}(x)$ for $\pi_{W_{v}^{+}}$the usual projection from $\mathbb{R}^{m}$ to $W_{v}^{+}$. $\Pi_{W_{v}^{-}}$is defined similarly.

Suppose $T_{4}<0$. Then $\left|T_{2}\right|=\left|T_{1}\right|+\left|T_{3}\right|+\left|T_{4}\right|$. Writing the loop as

$$
\begin{aligned}
e & =\mathbf{T}_{2} V_{1} \mathbf{T}_{3} U_{2} \mathbf{T}_{4} V_{2} \mathbf{T}_{1} U_{1} \\
& =\left(\mathbf{T}_{2} V_{1} \mathbf{T}_{2}^{-1}\right)\left(\mathbf{T}_{2} \mathbf{T}_{3} U_{2} \mathbf{T}_{3}^{-1} \mathbf{T}_{2}^{-1}\right)\left(\mathbf{T}_{2} \mathbf{T}_{3} \mathbf{T}_{4} V_{2} \mathbf{T}_{1}\right) U_{1},
\end{aligned}
$$


we see that only in the first bracket do we have a coordinate of size $e^{\left|T_{2}\right|}$. So $T_{4}>0$, and again by (iv) in the definition of quadrilateral, we conclude that for $i=1,2$, $d\left(e, \Pi_{W_{v}^{+}}\left(U_{i}\right)\right) \geq 1, d\left(e, \Pi_{W_{v}^{-}}\left(V_{i}\right)\right) \geq 1$.

Proof of Lemma 4.1.8 Summing the $\mathbb{R}$ coordinates we see that $r_{0}+r_{2}=r_{1}+r_{3}$. The identity word can be written as

$$
\begin{aligned}
e & =\left(r_{0}, 0\right) u_{0}\left(-r_{1}, 0\right) u_{1}\left(r_{2}, 0\right) u_{2}\left(-r_{3}, 0\right) u_{3} \\
& =\left(\left(r_{0}, 0\right) u_{0}\left(-r_{0}, 0\right)\right)\left(\left(r_{0}-r_{1}, 0\right) u_{1}\left(r_{1}-r_{0}, 0\right)\right)\left(\left(r_{3}, 0\right) u_{2}\left(-r_{3}, 0\right)\right) u_{3} .
\end{aligned}
$$

We see that $\left|r_{0}-r_{3}\right| \leq d\left(e, u_{0}\right)+d\left(e, u_{2}\right)$, and $\left|r_{0}-r_{1}\right| \leq d\left(e, u_{1}\right)+d\left(e, u_{3}\right)$ by comparing the $W^{+}$and $W^{-}$coordinates.

Similarly by looking at the word starting from $\left(-r_{1}, 0\right)$ we have

$$
\begin{aligned}
e & =\left(-r_{1}, 0\right) u_{1}\left(r_{2}, 0\right) u_{2}\left(-r_{3}, 0\right) u_{3}\left(r_{0}, 0\right) u_{0} \\
& =\left(\left(-r_{1}, 0\right) u_{1}\left(r_{1}, 0\right)\right)\left(\left(-r_{1}+r_{2}, 0\right) u_{2}\left(-r_{2}+r_{1}, 0\right)\right)\left(\left(-r_{0}, 0\right) u_{3}\left(r_{0}, 0\right)\right) u_{0}
\end{aligned}
$$

which gives us that $\left|r_{1}-r_{0}\right| \leq d\left(e, u_{1}\right)+d\left(e, u_{3}\right)$, and $\left|r_{2}-r_{1}\right| \leq d\left(e, u_{2}\right)+d\left(e, u_{0}\right)$. We obtain the desired claim by writing the word starting at $\left(r_{2}, 0\right)$ and $\left(-r_{3}, 0\right)$ and argue similarly as above.

Proof of Lemma 4.2.1 Equip the set $A \times B$ with the product measure $\mu=\mu_{\alpha} \times \mu_{\beta}$. The measure of the set $R=\{(a, b): a \sim b\}$ is therefore $\mu(R)=\int_{A} \mu_{\beta}\left(B_{a}\right) d \mu_{\alpha}=$ $\int_{B} \mu_{\alpha}\left(A_{b}\right) d \mu_{\beta}$. Hence

$$
\frac{1}{M_{B}} \frac{\mu(R)}{\mu_{\beta}(B)} \leq \mu_{\alpha}\left(A_{b}\right)_{\min }, \quad \mu_{\beta}\left(B_{a}\right)_{\max } \leq \frac{\mu(R)}{\mu_{\alpha}(A)} M_{A} .
$$

Let $\chi$ be the characteristic function of the set $\left\{(a, b): a \sim b, a \in A_{s}\right\}$. Then

$$
\begin{aligned}
\int_{B}\left(\int_{A_{b}} \chi d \mu_{\alpha}\right) d \mu_{\beta}=\int_{A}\left(\int_{B_{a}} \chi d \mu_{\beta}\right) d \mu_{\alpha} & =\int_{A_{s}} \mu_{\beta}\left(B_{a}\right) d \mu_{\alpha} \\
& \leq s \mu_{\alpha}(A) \mu_{\beta}\left(B_{a}\right)_{\max }, \\
\int_{B}\left(\int_{A_{b}} \chi d \mu_{\alpha}\right) d \mu_{\beta} \geq \int_{B^{s, t}}\left(\int_{A_{b}} \chi d \mu_{\alpha}\right) d \mu_{\beta} & \geq t \int_{B^{s, t}} \mu_{\alpha}\left(A_{b}\right) d \mu_{\beta} \\
& \geq t \mu_{\alpha}\left(A_{b}\right)_{\min } \mu_{\beta}\left(B^{s, t}\right) .
\end{aligned}
$$

Therefore

$$
\mu_{\beta}\left(B^{s, t}\right) \leq \frac{s \mu_{\alpha}(A) \mu_{\beta}\left(B_{a}\right)_{\max }}{t \mu_{\alpha}\left(A_{b}\right)_{\min }} \leq \frac{s}{t} M_{A} M_{B} \mu_{\beta}(B),
$$

where the last inequality comes from Equation (18). 


\section{References}

[1] L Auslander, An exposition of the structure of solvmanifolds, I: Algebraic theory, Bull. Amer. Math. Soc. 79 (1973) 227-261 MR0486307

[2] M R Bridson, S M Gersten, The optimal isoperimetric inequality for torus bundles over the circle, Quart. J. Math. Oxford Ser. (2) 47 (1996) 1-23 MR1380947

[3] T Dymarz, Large scale geometry of certain solvable groups, Geom. Funct. Anal. 19 (2010) 1650-1687 MR2594617

[4] T Dymarz, I Peng, Bilipschitz maps of boundaries of certain negatively curved homogeneous spaces, Geom. Dedicata 152 (2011) 129-145

[5] A Dyubina, Instability of the virtual solvability and the property of being virtually torsion-free for quasi-isometric groups, Internat. Math. Res. Notices (2000) 1097-1101 MR1800990

[6] A Eskin, D Fisher, K Whyte, Quasi-isometries and rigidity of solvable groups, Pure Appl. Math. Q. 3 (2007) 927-947 MR2402598

[7] A Eskin, D Fisher, K Whyte, Coarse differentiation of quasi-isometries I: Spaces not quasi-isometric to Cayley graphs arXiv:math/0607207

[8] A Eskin, D Fisher, K Whyte, Coarse differentiation of quasi-isometries II: Rigidity for Sol and Lamplighter groups arXiv:0706.0940

[9] Y Guivarc'h, Sur la loi des grands nombres et le rayon spectral d'une marche aléatoire, from: "Conference on Random Walks (Kleebach, 1979)", Astérisque 74, Soc. Math. France, Paris (1980) 47-98, 3 MR588157 In French

[10] D V Osin, Exponential radicals of solvable Lie groups, J. Algebra 248 (2002) 790-805 MR1882124

[11] I Peng, Coarse differentiation and quasi-isometries of a class of solvable Lie groups II, Geom. Topol. 15 (2011) 1927-1981

Department of Mathematics, Indiana University

831 E 3rd St, Bloomington IN 47401, USA

ipeng@indiana.edu

Proposed: Benson Farb

Received: 13 April 2009

Seconded: Danny Calegari, Martin R Bridson

Revised: 3 August 2011 